Pacific

Journal of

Mathematics

THE SPECTRUM OF AN INTEGRAL OPERATOR IN WEIGHTED $L_{2}$ SPACES

Mourad E.H. Ismail and Plamen C. Simeonov

Volume $198 \quad$ No. 2

April 2001 


\title{
THE SPECTRUM OF AN INTEGRAL OPERATOR IN WEIGHTED $L_{2}$ SPACES
}

\author{
Mourad E.H. Ismail and Plamen C. Simeonov
}

We find the spectrum of the inverse operator of the $q$ difference operator $D_{q, x} f(x)=(f(x)-f(q x)) /(x(1-q))$ on a family of weighted $L_{2}$ spaces. We show that the spectrum is discrete and the eigenvalues are the reciprocals of the zeros of an entire function. We also derive an expansion of the eigenfunctions of the $q$-difference operator and its inverse in terms of big $q$-Jacobi polynomials. This provides a $q$-analogue of the expansion of a plane wave in Jacobi polynomials. The coefficients are related to little $q$-Jacobi polynomials, which are described and proved to be orthogonal on the spectrum of the inverse operator. Explicit representations for the little $q$-Jacobi polynomials are given.

\section{Introduction.}

The $q$-difference operator $D_{q, x}$ is defined by

$$
D_{q, x} f(x):=\frac{f(x)-f(q x)}{x(1-q)} .
$$

We shall use the following notations for finite and infinite products:

$$
\begin{aligned}
(z ; q)_{0}:=1, \quad(z ; q)_{n} & :=\prod_{j=0}^{n-1}\left(1-q^{j} z\right), \quad n>0 \quad \text { or } \quad n=\infty, \\
\left(z_{1}, z_{2}, \ldots, z_{s} ; q\right)_{n} & :=\prod_{k=1}^{s}\left(z_{k} ; q\right)_{n}, \quad n \geq 0 \quad \text { or } \quad n=\infty .
\end{aligned}
$$

The infinite product is defined for $|q|<1$.

A basic hypergeometric series is defined by

$$
\begin{gathered}
{ }_{r} \phi_{s}\left(\begin{array}{c}
a_{1}, \ldots, a_{r} \\
b_{1}, \ldots, b_{s}
\end{array} \mid q, z\right)={ }_{r} \phi_{s}\left(a_{1}, \ldots, a_{r} ; b_{1}, \ldots, b_{s} ; q, z\right) \\
:=\sum_{n=0}^{\infty} \frac{\left(a_{1}, \ldots, a_{r} ; q\right)_{n}}{\left(q, b_{1}, \ldots, b_{s} ; q\right)_{n}} z^{n}\left((-1)^{n} q^{n(n-1) / 2}\right)^{1+s-r} .
\end{gathered}
$$


Let $\mathbf{t}=\left(t_{1}, t_{2}\right) \in \mathbf{R}^{2}$. The big $q$-Jacobi polynomials of Andrews and Askey, [2], are defined by

$$
p_{n}(x, \mathbf{t})=p_{n}\left(x ; t_{1}, t_{2}\right):={ }_{3} \phi_{2}\left(\begin{array}{c}
q^{-n}, a t_{1} t_{2} q^{n-1}, t_{1} x \\
t_{1}, a t_{1}
\end{array} \mid q, q\right), \quad 0<q<1
$$

and are orthogonal with respect to the measure $\mu(x, \mathbf{t}),[\mathbf{3}$, p. 594], [6], defined by

$$
\mu(x, \mathbf{t}):=\frac{\mu^{(a)}(x)}{\left(t_{1} x, t_{2} x ; q\right)_{\infty}},
$$

where for $a<0, \mu^{(a)}$ is the discrete probability measure

$$
\mu^{(a)}:=\sum_{n=0}^{\infty}\left[\frac{q^{n}}{(q, q / a ; q)_{n}(a ; q)_{\infty}} \delta_{q^{n}}+\frac{q^{n}}{(q, a q ; q)_{n}(1 / a ; q)_{\infty}} \delta_{a q^{n}}\right] .
$$

In (1.5), $\delta_{z}$ denotes the unit measure supported on $\{z\}$. The orthogonality relation is, $[\mathbf{3}],[\mathbf{6}]$,

$$
\int_{\mathbf{R}} p_{m}(x, \mathbf{t}) p_{n}(x, \mathbf{t}) d \mu(x, \mathbf{t})=\delta_{m, n} \xi_{n}(\mathbf{t}),
$$

where

$$
\xi_{n}(\mathbf{t})=\frac{\left(q, t_{2}, a t_{2}, a t_{1} t_{2} q^{n-1} ; q\right)_{n}\left(a t_{1} t_{2} q^{2 n} ; q\right)_{\infty}}{\left(t_{1}, a t_{1}, t_{2}, a t_{2} ; q\right)_{\infty}\left(t_{1}, a t_{1} ; q\right)_{n}}\left(-a t_{1}^{2}\right)^{n} q^{n(n-1) / 2} .
$$

Furthermore, (1.3), (1.6), (1.7), and the symmetry of $\mu$ in $t_{1}$ and $t_{2}$ imply the symmetry relation

$$
p_{n}\left(x ; t_{1}, t_{2}\right)=\frac{t_{1}^{n}\left(t_{2}, a t_{2} ; q\right)_{n}}{t_{2}^{n}\left(t_{1}, a t_{1} ; q\right)_{n}} p_{n}\left(x ; t_{2}, t_{1}\right) .
$$

We shall use the following $q$-analogue of the Chu-Vandermonde sum, $[4$, (II.6)],

$$
{ }_{2} \phi_{1}\left(q^{-n}, b ; c ; q, q\right)=\frac{(c / b ; q)_{n}}{(c ; q)_{n}} b^{n},
$$

and its special case $\left(b=t_{2} x, c=t_{2} q^{1-n} / t_{1}\right)$

$$
\left(t_{1} x ; q\right)_{n}=\left(t_{1} / t_{2} ; q\right)_{n 2} \phi_{1}\left(q^{-n}, t_{2} x ; t_{2} q^{1-n} / t_{1} ; q, q\right) .
$$

Letting $t_{1} \rightarrow \infty$ in (1.10) with $t_{2}=t$ we obtain

$$
t^{n} x^{n}=\sum_{j=0}^{n}\left[\begin{array}{l}
n \\
j
\end{array}\right]_{q}(-1)^{j} q^{\left(\begin{array}{l}
j \\
2
\end{array}\right)-j(n-1)}(t x ; q)_{j}
$$

where

$$
\left[\begin{array}{c}
n \\
j
\end{array}\right]_{q}:=\frac{(q ; q)_{n}}{(q ; q)_{j}(q ; q)_{n-j}}, \quad j=0, \ldots, n
$$


are the so called $q$-binomial coefficients.

We shall use Euler's identities, [6],

$$
\begin{aligned}
& e_{q}(z):=\frac{1}{(z ; q)_{\infty}}=\sum_{j=0}^{\infty} \frac{z^{j}}{(q ; q)_{j}}, \quad|z|<1, \\
& E_{q}(z):=(z ; q)_{\infty}=\sum_{j=0}^{\infty} \frac{q^{\left(\begin{array}{c}
j \\
2
\end{array}\right)}(-z)^{j}}{(q ; q)_{j}},
\end{aligned}
$$

and the terminating version of the $q$-binomial theorem, $[\mathbf{6}]$,

$$
(z ; q)_{n}=\sum_{j=0}^{n}\left[\begin{array}{l}
n \\
j
\end{array}\right]_{q} q^{\left(\begin{array}{l}
j \\
2
\end{array}\right)}(-z)^{j} .
$$

We shall also use the following identity

$$
\left(q^{1-n} / A ; q\right)_{k}=(-1 / A)^{k} q^{\left(\begin{array}{c}
k \\
2
\end{array}\right)+k(1-n)} \frac{(A ; q)_{n}}{(A ; q)_{n-k}} .
$$

The following theorem of H. Schwartz, [13], plays an important role in the spectral analysis in Section 2.

Theorem 1.1. Let $\left\{p_{n, \nu}(x)\right\}$ be a family of monic polynomials generated by

$$
\begin{aligned}
p_{0, \nu}(x) & =1, \quad p_{1, \nu}(x)=x+B_{\nu}, \\
p_{n+1, \nu}(x) & =\left(x+B_{n+\nu}\right) p_{n, \nu}(x)+C_{n+\nu} p_{n-1, \nu}(x), \quad n \geq 1 .
\end{aligned}
$$

If both

$$
\sum_{n=0}^{\infty}\left|B_{n+\nu}-\beta\right|<\infty \quad \text { and } \quad \sum_{n=0}^{\infty}\left|C_{n+\nu}\right|<\infty
$$

hold, then $x^{n} p_{n, \nu}(-\beta+1 / x)$ converges uniformly on compact subsets of the complex plane to an entire function of $x$.

The paper is organized in six sections. In Section 2, we study the eigenfunctions of the right inverse operator of $D_{q, x}$ on the space $L_{2}(\mu(\cdot, q \mathbf{t}))$. This right inverse operator will be denoted by $\mathcal{T}_{\mathbf{t}}$. The operator $\mathcal{T}_{\mathbf{t}}$ is defined first on the big $q$-Jacobi polynomials and then extended by linearity to $L_{2}(\mu(\cdot, q \mathbf{t}))$, in which the big $q$-Jacobi polynomials are complete. The operator $\mathcal{T}_{\mathbf{t}}$ is also a discrete integral operator. The rest of the paper is devoted to the study of the properties of $\mathcal{T}_{\mathbf{t}}$ and its eigenfunctions. It turns out that the matrix representation of $\mathcal{T}_{\mathbf{t}}$ in the basis formed by the big $q$-Jacobi polynomials is tridiagonal. This gives a three-term recurrence relation for the coefficients in the expansion of the eigenfunctions of $\mathcal{T}_{\mathbf{t}}$ in big $q$-Jacobi polynomials.

In Section 3, we find the polynomial solution of the recurrence relation for the coefficients in the expansion of the eigenfunctions of the operator $\mathcal{T}_{\mathbf{t}}$ 
from Section 2. The recurrence relation is identified with that of the little $q$-Jacobi polynomials.

In Section 4, we find the expansion of the formal eigenfunctions of the operator $D_{q, x}$ on the space $L_{2}(\mu(\cdot, \mathbf{t}))$ in terms of $\left\{p_{n}(x, \mathbf{t})\right\}$. The first coefficient of the expansion is used to determine the spectrum of the inverse operator $\mathcal{T}_{\mathbf{t}}$. This eigenfunction expansion gives a discrete $q$-analogue (on a $q$-linear lattice) of the well-known expansion of a plane wave $\exp (i \lambda x)$ in Jacobi polynomials.

Section 5 contains asymptotic properties of the orthogonal polynomials found in Section 3 and a formula for the Stieltjes transform of the measure of orthogonality. We prove that the measure of orthogonality is purely discrete and we identify the location of its masses with the discrete zero-set of an entire function and show how it is related to the spectrum of the operator $\mathcal{T}_{\mathrm{t}}$.

The paper concludes with Section 6, where we find the connection coefficients between families of big $q$-Jacobi polynomials corresponding to different values of the parameter $\mathbf{t}$.

\section{The Spectral Analysis.}

Let $\mathbf{t}=\left(t_{1}, t_{2}\right)$. By $q \mathbf{t}$ we shall denote the pair $\left(q t_{1}, q t_{2}\right)$. The Hilbert space $L_{2}(\mu(\cdot, \mathbf{t}))$ is defined through the standard dot-product

$$
\langle f, g\rangle=\int_{\mathbf{R}} f(x) \overline{g(x)} d \mu(x, \mathbf{t}),
$$

and

$$
L_{2}(\mu(\cdot, \mathbf{t}))=\left\{f:\|f\|_{L_{2}(\mu(\cdot, \mathbf{t}))}=\left(\int_{\mathbf{R}}|f(x)|^{2} d \mu(x, \mathbf{t})\right)^{1 / 2}<\infty\right\} .
$$

Let $\mathcal{T}_{\mathbf{t}}$ denote the right inverse operator of $D_{q, x}$ on $L_{2}(\mu(x, \mathbf{t}))$, that is, $\mathcal{T}_{\mathbf{t}}$ is a linear operator from $L_{2}(\mu(x, q \mathbf{t}))$ to $L_{2}(\mu(x, \mathbf{t}))$ such that $D_{q, x} \mathcal{T}_{\mathbf{t}}$ is the identity operator on the range of $D_{q, x}$ acting on $L_{2}(\mu(x, \mathbf{t}))$. From (1.3) and (1.1) we find

$$
\begin{aligned}
D_{q, x} p_{n}(x, \mathbf{t}) & =\frac{t_{1} q^{1-n}\left(1-q^{n}\right)\left(1-a t_{1} t_{2} q^{n-1}\right)}{(1-q)\left(1-t_{1}\right)\left(1-a t_{1}\right)} p_{n-1}(x, q \mathbf{t}) \\
& =: \sigma_{n}(\mathbf{t}) p_{n-1}(x, q \mathbf{t}) .
\end{aligned}
$$

Thus we require $\mathcal{T}_{\mathbf{t}}$ to satisfy

$$
\mathcal{T}_{\mathbf{t}} g(x) \sim \sum_{n=0}^{\infty}\left(g_{n} / \sigma_{n+1}(\mathbf{t})\right) p_{n+1}(x, \mathbf{t})
$$


if

$$
g(x) \sim \sum_{n=0}^{\infty} g_{n} p_{n}(x, q \mathbf{t})
$$

The operator $\mathcal{T}_{\mathbf{t}}$ can be expressed as an integral operator as well. From the orthogonality relation (1.6) we get

$$
g_{n}=\frac{1}{\xi_{n}(q \mathbf{t})} \int_{\mathbf{R}} g(u) p_{n}(u, q \mathbf{t}) d \mu(u, q \mathbf{t}) .
$$

Substituting in (2.2) and formally interchanging the order of integration and summation we get

$$
\mathcal{T}_{\mathbf{t}} g(x)=\int_{\mathbf{R}} g(u)\left\{\sum_{n=0}^{\infty} \frac{p_{n}(u, q \mathbf{t}) p_{n+1}(x, \mathbf{t})}{\xi_{n}(q \mathbf{t}) \sigma_{n+1}(\mathbf{t})}\right\} d \mu(u, q \mathbf{t}) .
$$

The sum in (2.3) is the kernel of the integral operator $\mathcal{T}_{\mathbf{t}}$.

We now consider the eigenvalue problem for the operator $\mathcal{T}_{\mathbf{t}}$, namely

$$
\mathcal{T}_{\mathbf{t}} g=\lambda g, \quad g(x)=\sum_{n=0}^{\infty} a_{n}(\lambda, \mathbf{t}) p_{n}(x, \mathbf{t}),
$$

with $g \in L_{2}(\mu(\cdot, \mathbf{t})) \cap L_{2}(\mu(\cdot, q \mathbf{t}))$. The function $g$ can be expanded in terms of the polynomials $\left\{p_{n}(x, \mathbf{t})\right\}$ since they are dense in $L_{2}(\mu(\cdot, \mathbf{t}))$. Furthermore, $(2.2)$ implies $a_{0}(\lambda, \mathbf{t})=0$ since $g$ is in the range of $\mathcal{T}_{\mathbf{t}}$.

We will need a connection coefficient formula of the form

$$
p_{n}(x, \mathbf{t})=\sum_{j=0}^{n} c_{n, j}(\mathbf{t}) p_{j}(x, q \mathbf{t}) .
$$

Such formula we can get using a simple duality theorem, [15, Theorem 2.5]. Let $\mu$ be a measure, $w$ and $\rho$, weight functions, and $\left\{p_{n}\right\}$ and $\left\{q_{n}\right\}$, polynomials orthogonal with respect to $w \mu$ and $\rho \mu$, respectively. Let $\alpha_{n}=$ $\int\left|p_{n}\right|^{2} w d \mu$ and $\beta_{n}=\int\left|q_{n}\right|^{2} \rho d \mu$. If

$$
w(x) p_{n}(x) \sim \rho(x) \sum_{j=n}^{\infty} c_{n, j} q_{j}(x), \quad \text { then } \quad q_{n}(x)=\sum_{k=0}^{n}\left(\beta_{n} / \alpha_{k}\right) c_{k, n} p_{k}(x) .
$$

Indeed, if $q_{n}=\sum_{k=0}^{n} d_{n, k} p_{k}$, then $d_{n, k}=\left(1 / \alpha_{k}\right) \int q_{n} p_{k} w d \mu=\left(\beta_{n} / \alpha_{k}\right) c_{k, n}$. Moreover, if $w / \rho$ is a polynomial of degree $s$, then $c_{n, j}=0$ for $j>n+s$. In our case with $w(x)=1 /\left(x q t_{1}, x q t_{2} ; q\right)_{\infty}, \rho(x)=1 /\left(x t_{1}, x t_{2} ; q\right)_{\infty}$, and $\mu=\mu^{(a)}$ we have $w(x) / \rho(x)=\left(1-t_{1} x\right)\left(1-t_{2} x\right)$. Thus

$$
p_{n}(x, \mathbf{t})=\sum_{m=n-2}^{n} c_{m, n}(\mathbf{t})\left(\xi_{n}(\mathbf{t}) / \xi_{m}(q \mathbf{t})\right) p_{m}(x, q \mathbf{t}), \quad n \geq 0
$$


where the coefficients $\left\{c_{m, n}(\mathbf{t})\right\}$ satisfy the equation

$$
\left(1-t_{1} x\right)\left(1-t_{2} x\right) p_{n}(x, q \mathbf{t})=\sum_{k=n}^{n+2} c_{n, k}(\mathbf{t}) p_{k}(x, \mathbf{t}), \quad n \geq 0 .
$$

The coefficients in (2.6) can be computed explicitly. Comparing the coefficients of $x^{n+2}$ in (2.6) we get

$$
\begin{aligned}
c_{n, n+2}(\mathbf{t}) & =\frac{\left(q^{-n}, a t_{1} t_{2} q^{n+1} ; q\right)_{n}}{\left(q^{-n-2}, a t_{1} t_{2} q^{n+1} ; q\right)_{n+2}} \frac{\left(t_{1}, a t_{1}, q ; q\right)_{n+2}}{\left(q t_{1}, a q t_{1}, q ; q\right)_{n}} \frac{q^{n}\left(-q t_{1}\right)^{n} q^{\left(\begin{array}{c}
n \\
2
\end{array}\right)}}{q^{n+2}\left(-t_{1}\right)^{n+2} q^{\left(\begin{array}{c}
n+2 \\
2
\end{array}\right)}} t_{1} t_{2} \\
& =\frac{\left(1-t_{1}\right)\left(1-a t_{1}\right)\left(1-t_{1} q^{n+1}\right)\left(1-a t_{1} q^{n+1}\right)}{\left(1-a t_{1} t_{2} q^{2 n+1}\right)\left(1-a t_{1} t_{2} q^{2 n+2}\right)}\left(t_{2} / t_{1}\right) q^{n} .
\end{aligned}
$$

From (1.3) and (1.8) we have $p_{m}\left(1 / t_{1} ; t_{1}, t_{2}\right)=1$ and

$$
p_{m}\left(1 / t_{2} ; t_{1}, t_{2}\right)=t_{1}^{m}\left(t_{2}, a t_{2} ; q\right)_{m} /\left(t_{2}^{m}\left(t_{1}, a t_{1} ; q\right)_{m}\right) .
$$

Substituting in (2.6) $x$ first by $1 / t_{1}$ and then by $1 / t_{2}$ we obtain

$$
\begin{gathered}
c_{n, n}(\mathbf{t})+c_{n, n+1}(\mathbf{t})+c_{n, n+2}(\mathbf{t})=0, \\
c_{n, n}(\mathbf{t})+\alpha_{n} c_{n, n+1}(\mathbf{t})+\alpha_{n} \alpha_{n+1} c_{n, n+2}(\mathbf{t})=0,
\end{gathered}
$$

where

$$
\alpha_{n}:=\frac{t_{1}\left(1-t_{2} q^{n}\right)\left(1-a t_{2} q^{n}\right)}{t_{2}\left(1-t_{1} q^{n}\right)\left(1-a t_{1} q^{n}\right)} .
$$

From (2.8) and (2.9) we obtain

$$
\begin{aligned}
c_{n, n}(\mathbf{t}) & =\frac{\alpha_{n}\left(\alpha_{n+1}-1\right)}{\alpha_{n}-1} c_{n, n+2}(\mathbf{t}) \\
& =\frac{\left(1-t_{1}\right)\left(1-a t_{1}\right)\left(1-t_{2} q^{n}\right)\left(1-a t_{2} q^{n}\right)}{\left(1-a t_{1} t_{2} q^{2 n}\right)\left(1-a t_{1} t_{2} q^{2 n+1}\right)} q^{n} .
\end{aligned}
$$

Applying $D_{q, x}$ to both sides of (2.4) and using (2.1) and (2.5) we obtain

$$
\begin{aligned}
D_{q, x}(\lambda g(x)) & =\lambda \sum_{n=1}^{\infty} a_{n}(\lambda, \mathbf{t}) \sigma_{n}(\mathbf{t}) p_{n-1}(x, q \mathbf{t})=g(x) \\
& =\sum_{m=1}^{\infty} a_{m}(\lambda, \mathbf{t}) p_{m}(x, \mathbf{t}) \\
& =\sum_{m=1}^{\infty} a_{m}(\lambda, \mathbf{t}) \sum_{j=m-2}^{m}\left(\xi_{m}(\mathbf{t}) / \xi_{j}(q \mathbf{t})\right) c_{j, m}(\mathbf{t}) p_{j}(x, q \mathbf{t}) .
\end{aligned}
$$


The above identity implies that the coefficients $\left\{a_{n}(\lambda, \mathbf{t})\right\}$ are generated by $(2.11)$

$$
\lambda a_{n}(\lambda, \mathbf{t}) \sigma_{n}(\mathbf{t})=\sum_{m=n-1}^{n+1}\left(\xi_{m}(\mathbf{t}) / \xi_{n-1}(q \mathbf{t})\right) c_{n-1, m}(\mathbf{t}) a_{m}(\lambda, \mathbf{t}), \quad n \geq 1,
$$

$a_{0}(\lambda, \mathbf{t})=0$ and $a_{1}(\lambda, \mathbf{t}) \neq 0$ is arbitrary. Formula (2.11) and the initial conditions show that $a_{n}(\lambda, \mathbf{t}) / a_{1}(\lambda, \mathbf{t})$ is a polynomial of degree $n-1$. We set

$$
\tilde{a}_{m-1}(\lambda, \mathbf{t}):=\xi_{m}(\mathbf{t}) a_{m}(\lambda, \mathbf{t}) /\left(\xi_{1}(\mathbf{t}) a_{1}(\lambda, \mathbf{t})\right), \quad m \geq 1,
$$

$\tilde{a}_{-1}(\lambda, \mathbf{t}):=0$ and $c_{n, m}(\mathbf{t}):=\left(1-t_{1}\right)\left(1-a t_{1}\right) \tilde{c}_{n, m}(\mathbf{t})$. Since

$$
\frac{\sigma_{n}(\mathbf{t}) \xi_{n-1}(q \mathbf{t})}{\xi_{n}(\mathbf{t})}=\frac{\left(1-t_{1}\right)\left(1-a t_{1}\right)}{-a t_{1}(1-q)},
$$

formula (2.11) can be written in the form

$$
\lambda \tilde{a}_{n}(\lambda, \mathbf{t})=-a t_{1}(1-q) \sum_{m=n}^{n+2} \tilde{c}_{n, m}(\mathbf{t}) \tilde{a}_{m-1}(\lambda, \mathbf{t}), \quad n \geq 0 .
$$

In terms of the variable $\eta$ and the functions $\tilde{b}_{m}(\eta, \mathbf{t})$ defined by

$$
\eta:=-\lambda /\left(a t_{1}(1-q)\right) \quad \text { and } \quad \tilde{b}_{m}(\eta, \mathbf{t}):=\tilde{a}_{m}\left(-a t_{1}(1-q) \eta, \mathbf{t}\right),
$$

formula (2.12) can be written in the form

$$
\eta \tilde{b}_{n}(\eta, \mathbf{t})=\sum_{m=n}^{n+2} \tilde{c}_{n, m}(\mathbf{t}) \tilde{b}_{m-1}(\eta, \mathbf{t}), \quad n \geq 0
$$

with the initial conditions

$$
\tilde{b}_{-1}(\eta, \mathbf{t})=0, \quad \tilde{b}_{0}(\eta, \mathbf{t})=1 .
$$

From (2.10), (2.7) and (2.8) for the coefficients we obtain

$$
\begin{gathered}
\tilde{c}_{n, n}=\tilde{c}_{n, n}(\mathbf{t})=\frac{\left(1-t_{2} q^{n}\right)\left(1-a t_{2} q^{n}\right)}{\left(1-a t_{1} t_{2} q^{2 n}\right)\left(1-a t_{1} t_{2} q^{2 n+1}\right)} q^{n}, \\
\tilde{c}_{n, n+2}=\tilde{c}_{n, n+2}(\mathbf{t})=\frac{\left(1-t_{1} q^{n+1}\right)\left(1-a t_{1} q^{n+1}\right) t_{2}}{\left(1-a t_{1} t_{2} q^{2 n+1}\right)\left(1-a t_{1} t_{2} q^{2 n+2}\right) t_{1}} q^{n}, \\
\tilde{c}_{n, n+1}=\tilde{c}_{n, n+1}(\mathbf{t})=-\left(\tilde{c}_{n, n}(\mathbf{t})+\tilde{c}_{n, n+2}(\mathbf{t})\right) .
\end{gathered}
$$

It is convenient to have (2.13) written in monic form. Let

$$
\tilde{b}_{m}(\eta, \mathbf{t}):=G_{m}(\mathbf{t}) b_{m}(\eta, \mathbf{t}) .
$$


From (2.13) we have

$$
\begin{aligned}
\eta b_{n}(\eta, \mathbf{t})= & \frac{\tilde{c}_{n, n} G_{n-1}(\mathbf{t})}{G_{n}(\mathbf{t})} b_{n-1}(\eta, \mathbf{t})+\tilde{c}_{n, n+1} b_{n}(\eta, \mathbf{t}) \\
& +\frac{\tilde{c}_{n, n+2} G_{n+1}(\mathbf{t})}{G_{n}(\mathbf{t})} b_{n+1}(\eta, \mathbf{t}), \quad n \geq 0,
\end{aligned}
$$

which is a monic equation if the coefficient of $b_{n+1}(\eta, \mathbf{t})$ is 1 , that is, if $G_{n+1}(\mathbf{t})=G_{n}(\mathbf{t}) / \tilde{c}_{n, n+2}$. In this case

$$
G_{n}(\mathbf{t})=G_{0}(\mathbf{t}) / \prod_{j=0}^{n-1} \tilde{c}_{j, j+2}, \quad n \geq 1, \quad G_{0}(\mathbf{t}) \neq 0,
$$

and (2.13) takes the form

$$
\eta b_{n}(\eta, \mathbf{t})=b_{n+1}(\eta, \mathbf{t})+\tilde{c}_{n, n+1} b_{n}(\eta, \mathbf{t})+\tilde{c}_{n, n} \tilde{c}_{n-1, n+1} b_{n-1}(\eta, \mathbf{t}), \quad n \geq 0 .
$$

We set $b_{0}(\eta, \mathbf{t})=G_{0}(\mathbf{t})=1$ and $b_{-1}(\eta, \mathbf{t})=0$. When the coefficients in (2.17) are written explicitly in terms of $t_{1}, t_{2}$, and $n$, we obtain

$$
\begin{gathered}
b_{n+1}(\eta, \mathbf{t})=\left(\eta+\frac{\left(1-t_{2} q^{n}\right)\left(1-a t_{2} q^{n}\right)}{\left(1-a t_{1} t_{2} q^{2 n}\right)\left(1-a t_{1} t_{2} q^{2 n+1}\right)} q^{n}\right. \\
\left.+\frac{\left(1-t_{1} q^{n+1}\right)\left(1-a t_{1} q^{n+1}\right) t_{2}}{\left(1-a t_{1} t_{2} q^{2 n+1}\right)\left(1-a t_{1} t_{2} q^{2 n+2}\right) t_{1}} q^{n}\right) b_{n}(\eta, \mathbf{t}) \\
-\frac{\left(1-t_{1} q^{n}\right)\left(1-a t_{1} q^{n}\right)\left(1-t_{2} q^{n}\right)\left(1-a t_{2} q^{n}\right) t_{2}}{\left(1-a t_{1} t_{2} q^{2 n-1}\right)\left(1-a t_{1} t_{2} q^{2 n}\right)^{2}\left(1-a t_{1} t_{2} q^{2 n+1}\right) t_{1}} q^{2 n-1} b_{n-1}(\eta, \mathbf{t}) .
\end{gathered}
$$

We now apply Schwartz's theorem (Theorem 1.1). With $B_{n}(\mathbf{t})=$ $-t_{1} \tilde{c}_{n, n+1}(\mathbf{t}), C_{n}(\mathbf{t})=-t_{1}^{2} \tilde{c}_{n, n}(\mathbf{t}) \tilde{c}_{n-1, n+1}(\mathbf{t})$, and $\hat{b}_{n}(\eta, \mathbf{t})=t_{1}^{n} b_{n}\left(\eta / t_{1}, \mathbf{t}\right)$, (2.18) takes the form

$$
\hat{b}_{n+1}(\eta, \mathbf{t})=\left(\eta+B_{n}(\mathbf{t})\right) \hat{b}_{n}(\eta, \mathbf{t})+C_{n}(\mathbf{t}) \hat{b}_{n-1}(\eta, \mathbf{t}), \quad n \geq 0 .
$$

Furthermore, by (2.14)-(2.16) we have $B_{n}\left(q^{\nu} \mathbf{t}\right)=B_{n+\nu}(\mathbf{t}), C_{n}\left(q^{\nu} \mathbf{t}\right)=$ $C_{n+\nu}(\mathbf{t})$,

$$
\sum_{n=0}^{\infty}\left|B_{n}(\mathbf{t})\right|<\infty, \quad \text { and } \quad \sum_{n=0}^{\infty}\left|C_{n}(\mathbf{t})\right|<\infty .
$$

Then by Theorem 1.1, $\eta^{n} \hat{b}_{n}(1 / \eta, \mathbf{t})=\left(t_{1} \eta\right)^{n} b_{n}\left(1 /\left(t_{1} \eta\right), \mathbf{t}\right)$, or equivalently, $\eta^{n} b_{n}(1 / \eta, \mathbf{t})$ converges locally uniformly in the complex plane to an entire function of $\eta$. 


\section{The Polynomial Solution of the Recurrence Equation for the Coefficients.}

In this section we solve recurrence equation (2.18):

$$
b_{n+1}(\eta, \mathbf{t})=\left(\eta+\beta_{n} q^{n}\right) b_{n}(\eta, \mathbf{t})-\gamma_{n} q^{2 n-1} b_{n-1}(\eta, \mathbf{t}), \quad n \geq 0,
$$

with $b_{-1}(\eta, \mathbf{t})=0, b_{0}(\eta, \mathbf{t})=1$, and

$$
\beta_{n}=\frac{\left(1-t_{2} q^{n}\right)\left(1-a t_{2} q^{n}\right)}{\left(1-a t_{1} t_{2} q^{2 n}\right)\left(1-a t_{1} t_{2} q^{2 n+1}\right)}+\frac{\left(1-t_{1} q^{n+1}\right)\left(1-a t_{1} q^{n+1}\right) t_{2}}{\left(1-a t_{1} t_{2} q^{2 n+1}\right)\left(1-a t_{1} t_{2} q^{2 n+2}\right) t_{1}},
$$

$$
\gamma_{n}=\frac{\left(1-t_{1} q^{n}\right)\left(1-a t_{1} q^{n}\right)\left(1-t_{2} q^{n}\right)\left(1-a t_{2} q^{n}\right) t_{2}}{\left(1-a t_{1} t_{2} q^{2 n-1}\right)\left(1-a t_{1} t_{2} q^{2 n}\right)^{2}\left(1-a t_{1} t_{2} q^{2 n+1}\right) t_{1}} .
$$

The coefficients $\beta_{n}$ can be simplified. We have

$$
\begin{aligned}
t_{1}\left(a t_{1} t_{2} q^{2 n} ; q\right)_{3} \beta_{n}= & t_{1}\left(1-t_{2} q^{n}\right)\left(1-a t_{2} q^{n}\right)\left(1-a t_{1} t_{2} q^{2 n+2}\right) \\
& +t_{2}\left(1-t_{1} q^{n+1}\right)\left(1-a t_{1} q^{n+1}\right)\left(1-a t_{1} t_{2} q^{2 n}\right) \\
= & t_{1}\left(1-(1+a) t_{2} q^{n}+a t_{2}^{2} q^{2 n}-a t_{1} t_{2} q^{2 n+2}\right. \\
& \left.+a(1+a) t_{1} t_{2}^{2} q^{3 n+2}-a^{2} t_{1} t_{2}^{3} q^{4 n+2}\right) \\
& +t_{2}\left(1-(1+a) t_{1} q^{n+1}+a t_{1}^{2} q^{2 n+2}-a t_{1} t_{2} q^{2 n}\right. \\
& \left.+a(1+a) t_{1}^{2} t_{2} q^{3 n+1}-a^{2} t_{1}^{3} t_{2} q^{4 n+2}\right) \\
= & \left(t_{1}+t_{2}\right)\left(1-a^{2} t_{1}^{2} t_{2}^{2} q^{4 n+2}\right)-(1+a) t_{1} t_{2} q^{n}(1+q) \\
& +a(1+a) t_{1}^{2} t_{2}^{2} q^{3 n+1}(1+q) \\
= & \left(1-a t_{1} t_{2} q^{2 n+1}\right)\left(\left(t_{1}+t_{2}\right)\left(1+a t_{1} t_{2} q^{2 n+1}\right)\right. \\
& \left.-(1+a)(1+q) t_{1} t_{2} q^{n}\right) .
\end{aligned}
$$

Therefore,

$$
\beta_{n}=\frac{\left(t_{1}+t_{2}\right)\left(1+a t_{1} t_{2} q^{2 n+1}\right)-(1+a)(1+q) t_{1} t_{2} q^{n}}{\left(1-a t_{1} t_{2} q^{2 n}\right)\left(1-a t_{1} t_{2} q^{2 n+2}\right) t_{1}} .
$$

Recurrence relation (3.1) can be identified with the recurrence relation of the associated little $q$-Jacobi polynomials, [5]. The latter work gets the little $q$-Jacobi polynomials as limiting cases of the associated big $q$-Jacobi polynomials and does not give an explicit representation for the polynomials. Theorem 4.1 below provides an explicit representation for the little $q$-Jacobi polynomials.

We shall use (3.1)-(3.4) to find the coefficient $\beta_{n, n-1}$ of $\eta^{n-1}$ in $b_{n}(\eta, \mathbf{t})$ and to guess the structure of the polynomials $b_{n}(\eta, \mathbf{t})$. From (3.1) we get 
$\beta_{n+1, n}=\beta_{n} q^{n}+\beta_{n, n-1}$ which implies $\beta_{n, n-1}=\sum_{j=0}^{n-1} \beta_{j} q^{j}$. From (3.4) we have

$$
\begin{aligned}
& a(1-q) t_{1} \beta_{j} q^{j}=a\left(t_{1}+t_{2}\right) q^{j}\left(\frac{1}{1-a t_{1} t_{2} q^{2 j}}-\frac{q}{1-a t_{1} t_{2} q^{2 j+2}}\right) \\
& -(1+a)\left(\frac{1}{1-a t_{1} t_{2} q^{2 j}}-\frac{1}{1-a t_{1} t_{2} q^{2 j+2}}\right)=: R\left(q^{j}\right)-R\left(q^{j+1}\right),
\end{aligned}
$$

where

$$
R(z)=\frac{a\left(t_{1}+t_{2}\right) z-(1+a)}{1-a t_{1} t_{2} z^{2}} .
$$

Then $\beta_{n, n-1}$ becomes a telescoping sum and we find

$$
\begin{aligned}
& a(1-q) t_{1} \beta_{n, n-1} \\
& =(R(1)+1)-\left(R\left(q^{n}\right)+1\right) \\
& =-\frac{a\left(1-t_{1}\right)\left(1-t_{2}\right)}{\left(1-a t_{1} t_{2}\right)}+\frac{a\left(1-t_{1} q^{n}\right)\left(1-t_{2} q^{n}\right)}{\left(1-a t_{1} t_{2} q^{2 n}\right)} \\
& =\frac{a\left(1-t_{1} q^{n}\right)\left(1-t_{2} q^{n}\right)}{\left(1-a t_{1} t_{2} q^{2 n}\right)}\left(1-\frac{\left(1-t_{1}\right)\left(1-t_{2}\right)\left(1-a t_{1} t_{2} q^{2 n}\right)}{\left(1-a t_{1} t_{2}\right)\left(1-t_{1} q^{n}\right)\left(1-t_{2} q^{n}\right)}\right) .
\end{aligned}
$$

The coefficient $\beta_{n, n-1}$ can be written in a form that resembles similar formulas for the Wimp polynomials from [16] and their $q$-analogue from [8]:

$$
\begin{aligned}
\beta_{n, n-1}=- & \frac{\left(1-q^{-n} / t_{1}\right)\left(1-q^{-n} / t_{2}\right)}{(1-q)\left(1-q^{-2 n} /\left(a t_{1} t_{2}\right)\right)}\left(a t_{1}\right)^{-1} \\
& \times\left(1+\frac{\left(q^{-1}, t_{1}, t_{2}, a t_{1} t_{2} q^{2 n} ; q\right)_{1} q}{\left(a t_{1} t_{2}, t_{1} q^{n}, t_{2} q^{n}, q ; q\right)_{1}}\right) .
\end{aligned}
$$

The analogue of these polynomials that solves (3.1) is defined below.

Theorem 3.1. The polynomials $b_{n}(\eta, \mathbf{t})$ defined by

$$
\begin{aligned}
b_{n}(\eta, \mathbf{t})= & \sum_{j=0}^{n} \frac{\left(q^{-n} / t_{1}, q^{-n} / t_{2} ; q\right)_{j}}{\left(q, q^{-2 n} /\left(a t_{1} t_{2}\right) ; q\right)_{j}}\left(-a t_{1}\right)^{-j} \eta^{n-j} \\
& \times{ }_{4} \phi_{3}\left(\begin{array}{c}
q^{-j}, t_{1}, t_{2}, a t_{1} t_{2} q^{2 n+1-j} \\
t_{1} q^{n+1-j}, t_{2} q^{n+1-j}, a t_{1} t_{2}
\end{array} \mid q, q\right), \quad n \geq 0
\end{aligned}
$$

are the solutions of the recurrence relation (3.1)-(3.4).

Proof. The proof is similar to the proof of Theorem 4.1 in [9]. Let $\tilde{b}_{n}(\eta, \mathbf{t})$ denote the polynomial on the right-hand side of (3.6). We shall demonstrate that $\tilde{b}_{n}(\eta, \mathbf{t})$ satisfies $(3.1)$. 
The polynomials $\tilde{b}_{n}(\eta, \mathbf{t})$ can be written in the form

$$
\begin{aligned}
\tilde{b}_{n}(\eta, \mathbf{t})= & \sum_{j=0}^{n} \eta^{n-j} \sum_{k=0}^{j} \frac{(-1)^{j}}{\left(a t_{1}\right)^{j}} \frac{\left(t_{1}, t_{2} ; q\right)_{k}}{\left(q, a t_{1} t_{2} ; q\right)_{k}} \\
& \times \frac{\left(q^{-n} / t_{1}, q^{-n} / t_{2} ; q\right)_{j}}{\left(t_{1} q^{n+1-j}, t_{2} q^{n+1-j} ; q\right)_{k}} \frac{\left(a t_{1} t_{2} q^{2 n+1-j} ; q\right)_{k}}{\left(q^{-2 n} /\left(a t_{1} t_{2}\right) ; q\right)_{j}} \frac{\left(q^{-j} ; q\right)_{k} q^{k}}{(q ; q)_{j}} \\
= & \sum_{j=0}^{n} \eta^{n-j} \sum_{k=0}^{j} \frac{(-1)^{j}}{\left(a t_{1}\right)^{j}} \frac{\left(t_{1}, t_{2} ; q\right)_{k}}{\left(q, a t_{1} t_{2} ; q\right)_{k}} a^{k} \frac{\left(q^{-n} / t_{1}, q^{-n} / t_{2} ; q\right)_{j-k}}{\left(q, q^{-2 n} /\left(a t_{1} t_{2}\right) ; q\right)_{j-k}} \\
= & : \sum_{j=0}^{n} \sum_{k=0}^{j} A_{k} B_{j-k}^{(n)}\left(-1 / t_{1}\right)^{j} a^{k-j} \eta^{n-j},
\end{aligned}
$$

where we applied formula (1.15) with $A=1 /\left(t_{1} q^{n}\right), 1 /\left(t_{2} q^{n}\right), 1 /\left(a t_{1} t_{2} q^{2 n}\right)$, and $q$, and we defined

$$
\begin{aligned}
A_{k} & :=\frac{\left(t_{1}, t_{2} ; q\right)_{k}}{\left(q, a t_{1} t_{2} ; q\right)_{k}}, \\
B_{s}^{(n)} & :=\frac{\left(q^{-n} / t_{1}, q^{-n} / t_{2} ; q\right)_{s}}{\left(q, q^{-2 n} /\left(a t_{1} t_{2}\right) ; q\right)_{s}} .
\end{aligned}
$$

We separate the leading term $\eta^{n+1}$ and write $\tilde{b}_{n+1}(\eta, \mathbf{t})$ in the form

$$
\begin{aligned}
\tilde{b}_{n+1}(\eta, \mathbf{t})= & \eta\left(\tilde{b}_{n}(\eta, \mathbf{t})-\sum_{j=1}^{n} \sum_{k=0}^{j} A_{k} B_{j-k}^{(n)} a^{k-j}\left(-1 / t_{1}\right)^{j} \eta^{n-j}\right) \\
& +\sum_{j=1}^{n+1} \sum_{k=0}^{j} A_{k} B_{j-k}^{(n+1)} a^{k-j}\left(-1 / t_{1}\right)^{j} \eta^{n+1-j} \\
= & \left(\eta+\beta_{n} q^{n}\right) \tilde{b}_{n}(\eta, \mathbf{t})-\tilde{r}_{n}(\eta, \mathbf{t}),
\end{aligned}
$$


where

$$
\begin{aligned}
\tilde{r}_{n}(\eta, \mathbf{t}):= & \sum_{j=1}^{n} \sum_{k=0}^{j} A_{k} B_{j-k}^{(n)} a^{k-j}\left(-1 / t_{1}\right)^{j} \eta^{n+1-j} \\
& -\sum_{j=1}^{n+1} \sum_{k=0}^{j} A_{k} B_{j-k}^{(n+1)} a^{k-j}\left(-1 / t_{1}\right)^{j} \eta^{n+1-j} \\
& +\beta_{n} q^{n} \sum_{j=0}^{n} \sum_{k=0}^{j} A_{k} B_{j-k}^{(n)} a^{k-j}\left(-1 / t_{1}\right)^{j} \eta^{n-j} \\
& \sum_{j=0}^{n-1} \sum_{k=0}^{j+1} A_{k} B_{j+1-k}^{(n)} a^{k-j-1}\left(-1 / t_{1}\right)^{j+1} \eta^{n-j} \\
& -\sum_{j=0}^{n} \sum_{k=0}^{j+1} A_{k} B_{j+1-k}^{(n+1)} a^{k-j-1}\left(-1 / t_{1}\right)^{j+1} \eta^{n-j} \\
& +\beta_{n} q^{n} \sum_{j=0}^{n} \sum_{k=0}^{j} A_{k} B_{j-k}^{(n)} a^{k-j}\left(-1 / t_{1}\right)^{j} \eta^{n-j} .
\end{aligned}
$$

The coefficient of $\eta^{n}$ in $\tilde{r}_{n}(\eta, \mathbf{t})$ equals

$$
\begin{aligned}
& \sum_{k=0}^{1} A_{k}\left(B_{1-k}^{(n)}-B_{1-k}^{(n+1)}\right) a^{k-1}\left(-1 / t_{1}\right)+\beta_{n} q^{n} \\
& =-\left\{B_{1}^{(n)}-B_{1}^{(n+1)}\right\} /\left(a t_{1}\right)+\beta_{n} q^{n}
\end{aligned}
$$

since $A_{0}=1$ and $B_{0}^{(n)}=1$. Furthermore,

$$
B_{1}^{(n)}-B_{1}^{(n+1)}=-\frac{a}{1-q}\left(\frac{\left(1-t_{1} q^{n}\right)\left(1-t_{2} q^{n}\right)}{1-a t_{1} t_{2} q^{2 n}}-\frac{\left(1-t_{1} q^{n+1}\right)\left(1-t_{2} q^{n+1}\right)}{1-a t_{1} t_{2} q^{2 n+2}}\right)
$$

and then

$$
\begin{aligned}
- & \left(a t_{1} t_{2} q^{2 n} ; q^{2}\right)_{2}((1-q) / a)\left\{B_{1}^{(n)}-B_{1}^{(n+1)}\right\} \\
= & \left(1-t_{1} q^{n}\right)\left(1-t_{2} q^{n}\right)\left(1-a t_{1} t_{2} q^{2 n+2}\right) \\
& -\left(1-t_{1} q^{n+1}\right)\left(1-t_{2} q^{n+1}\right)\left(1-a t_{1} t_{2} q^{2 n}\right) \\
= & -q^{n}\left(\left(t_{1}+t_{2}\right)(1-q)\left(1+a t_{1} t_{2} q^{2 n+1}\right)-(1+a)\left(1-q^{2}\right) t_{1} t_{2} q^{n}\right) .
\end{aligned}
$$

Hence in view of (3.4) we get

$$
-\left\{B_{1}^{(n)}-B_{1}^{(n+1)}\right\} /\left(a t_{1}\right)=-\beta_{n} q^{n},
$$

which shows that the coefficient of $\eta^{n}$ in $\tilde{r}_{n}(\eta, \mathbf{t})$ is zero. Then in (3.9) we can replace the lower bound of the range of $j$ by 1 and then replace $j$ by 
$j+1$. We obtain

$$
\begin{aligned}
\tilde{r}_{n}(\eta, \mathbf{t})= & \beta_{n} q^{n} \sum_{j=0}^{n-1} \sum_{k=0}^{j+1} A_{k} B_{j+1-k}^{(n)} a^{k-j-1}\left(-1 / t_{1}\right)^{j+1} \eta^{n-j-1} \\
& +\sum_{j=0}^{n-2} \sum_{k=0}^{j+2} A_{k} B_{j+2-k}^{(n)} a^{k-j-2}\left(-1 / t_{1}\right)^{j+2} \eta^{n-j-1} \\
& -\sum_{j=0}^{n-1} \sum_{k=0}^{j+2} A_{k} B_{j+2-k}^{(n+1)} a^{k-j-2}\left(-1 / t_{1}\right)^{j+2} \eta^{n-j-1} .
\end{aligned}
$$

Note that in (3.11) we can first separate the two constant terms, and then in the last two double sums we can replace the upper bound $j+2$ of the range of $k$ by $j+1$ using that $B_{j+2-k}^{(n)}-B_{j+2-k}^{(n+1)}=0$ if $k=j+2$. Then we can write

$$
\tilde{r}_{n}(\eta, \mathbf{t})=K_{n}+\sum_{j=0}^{n-2} \sum_{k=0}^{j+1} A_{k} a^{k-j-2}\left(-1 / t_{1}\right)^{j+2} \triangle_{j+2-k}^{(n)} \eta^{n-j-1},
$$

where

$$
\triangle_{s}^{(n)}=B_{s}^{(n)}-B_{s}^{(n+1)}-a t_{1} \beta_{n} q^{n} B_{s-1}^{(n)}, \quad s=1, \ldots, n,
$$

and $K_{n}$ is the constant term of $\tilde{r}_{n}(\eta, \mathbf{t})$.

From (3.10) we get $\triangle_{1}^{(n)}=0$.

For $s \in\{2, \ldots, n\}$ we have

$$
\begin{aligned}
\triangle_{s}^{(n)}= & \frac{\left(q^{-n} / t_{1}, q^{-n} / t_{2} ; q\right)_{s}}{\left(q, q^{-2 n} /\left(a t_{1} t_{2}\right) ; q\right)_{s}}-\frac{\left(q^{-n-1} / t_{1}, q^{-n-1} / t_{2} ; q\right)_{s}}{\left(q, q^{-2 n-2} /\left(a t_{1} t_{2}\right) ; q\right)_{s}} \\
& -\frac{q^{-n-1}}{t_{1} t_{2}} \frac{\left(\left(t_{1}+t_{2}\right)\left(1+q^{-2 n-1} /\left(a t_{1} t_{2}\right)\right)-(1+a)(1+q) q^{-n-1} / a\right)}{\left(1-q^{-2 n} /\left(a t_{1} t_{2}\right)\right)\left(1-q^{-2 n-2} /\left(a t_{1} t_{2}\right)\right)} \\
& \times \frac{\left(q^{-n} / t_{1}, q^{-n} / t_{2} ; q\right)_{s-1}}{\left(q, q^{-2 n} /\left(a t_{1} t_{2}\right) ; q\right)_{s-1}} \\
= & \frac{\left(q^{-n} / t_{1}, q^{-n} / t_{2} ; q\right)_{s-1}}{(q ; q)_{s}\left(q^{-2 n-2} /\left(a t_{1} t_{2}\right) ; q\right)_{s+2}\left(1-q^{-2 n} /\left(a t_{1} t_{2}\right)\right)} \\
& \times\left\{\left[\left(1-q^{-n-1+s} / t_{1}\right)\left(1-q^{-n-1+s} / t_{2}\right)\left(q^{-2 n-2} /\left(a t_{1} t_{2}\right) ; q\right)_{2}\right.\right. \\
& \left.-\left(1-q^{-n-1} / t_{1}\right)\left(1-q^{-n-1} / t_{2}\right)\left(q^{-2 n-2+s} /\left(a t_{1} t_{2}\right) ; q\right)_{2}\right] \\
& \times\left(1-q^{-2 n} /\left(a t_{1} t_{2}\right)\right)-\left(q^{-n-1} /\left(t_{1} t_{2}\right)\right)\left(1-q^{s}\right) \\
& \times\left(\left(t_{1}+t_{2}\right)\left(1+q^{-2 n-1} /\left(a t_{1} t_{2}\right)\right)-(1+a)(1+q) q^{-n-1} / a\right) \\
& \left.\times\left(1-q^{-2 n-1} /\left(a t_{1} t_{2}\right)\right)\left(1-q^{-2 n-1+s} /\left(a t_{1} t_{2}\right)\right)\right\} .
\end{aligned}
$$


We set $x=q^{-n-1} / t_{1}, y=q^{-n-1} / t_{2}, \alpha=q^{s}, \beta=1 / a, S=x+y$, and $P=x y$. Let $E_{1}$ and $E_{2}$ denote the expressions inside \{\} and [ ] in (3.14), respectively. Then

$$
\begin{aligned}
E_{1}= & E_{2}\left(1-q^{2} \beta P\right) \\
& -(1-\alpha)(S(1+q \beta P)-(1+\beta)(1+q) P)(1-q \beta P)(1-q \alpha \beta P)
\end{aligned}
$$

and

$$
\begin{aligned}
E_{2}= & (1-\alpha x)(1-\alpha y)(1-\beta P)(1-q \beta P) \\
& -(1-x)(1-y)(1-\alpha \beta P)(1-q \alpha \beta P) .
\end{aligned}
$$

We now simplify $E_{2}$. We have

$$
\begin{aligned}
E_{2}= & 1-\alpha S+\alpha^{2} P-(1+q) \beta P\left(1-\alpha S+\alpha^{2} P\right)+q \beta^{2} P^{2}\left(1-\alpha S+\alpha^{2} P\right) \\
& -1+S-P+(1+q) \alpha \beta P(1-S+P)-q \alpha^{2} \beta^{2} P^{2}(1-S+P) \\
= & (1-\alpha)[S-(1+\alpha) P-(1+q) \beta P \\
& \left.+(1+q) \alpha \beta P^{2}+q(1+\alpha) \beta^{2} P^{2}-q \alpha \beta^{2} P^{2} S\right] \\
= & (1-\alpha)\left[S\left(1-q \alpha \beta^{2} P^{2}\right)-(1+\alpha)\left(1-q \beta^{2} P\right) P-(1+q) \beta(1-\alpha P) P\right] .
\end{aligned}
$$

From (3.15) and the definition of $E_{1}$ and $E_{2}$ we obtain

$$
\begin{aligned}
E_{1} /(1-\alpha)= & S\left[\left(1-q \alpha \beta^{2} P^{2}\right)\left(1-q^{2} \beta P\right)-\left(1-q^{2} \beta^{2} P^{2}\right)(1-q \alpha \beta P)\right] \\
& -P\left[\left((1+\alpha)\left(1-q \beta^{2} P\right)+(1+q) \beta(1-\alpha P)\right)\left(1-q^{2} \beta P\right)\right. \\
& -(1+q)(1+\beta)(1-q \beta P)(1-q \alpha \beta P)] \\
= & : B_{1} S-B_{2} P,
\end{aligned}
$$

where $B_{1}$ and $B_{2}$ denote the expressions inside the brackets. We factor $B_{1}$ and $B_{2}$ :

$$
\begin{aligned}
B_{1} & =1-q \alpha \beta^{2} P^{2}-q^{2} \beta P+q^{3} \alpha \beta^{3} P^{3}-1+q^{2} \beta^{2} P^{2}+q \alpha \beta P-q^{3} \alpha \beta^{3} P^{3} \\
& =q \beta P(\alpha+q \beta P-q-\alpha \beta P)=q \beta(\alpha-q)(1-\beta P) P,
\end{aligned}
$$


(3.18)

$$
\begin{aligned}
B_{2}= & (1+\alpha+(1+q) \beta-\beta(q \beta(1+\alpha)+(1+q) \alpha) P)\left(1-q^{2} \beta P\right) \\
& -(1+q)(1+\beta)\left(1-q \beta(1+\alpha) P+q^{2} \alpha \beta^{2} P^{2}\right) \\
= & \alpha-q+\beta\left((1+\alpha)\left(q(1+q)(1+\beta)-q \beta-q^{2}\right)-(1+q)\left(\alpha+q^{2} \beta\right)\right) P \\
& +q^{2} \beta^{2}(q(1+\alpha) \beta+(1+q) \alpha-(1+q) \alpha(1+\beta)) P^{2} \\
= & \alpha-q+\beta\left((1+\alpha)\left(q+\beta q^{2}\right)-(1+q)\left(\alpha+\beta q^{2}\right)\right) P+q^{2}(q-\alpha) \beta^{3} P^{2} \\
= & -(q-\alpha)+\beta(q-\alpha)\left(1-\beta q^{2}\right) P+q^{2}(q-\alpha) \beta^{3} P^{2} \\
= & -(q-\alpha)(1-\beta P)\left(1+q^{2} \beta^{2} P\right) .
\end{aligned}
$$

From (3.16)-(3.18) we obtain

$$
\begin{aligned}
& \frac{E_{1}}{(1-\alpha)(q-\alpha)(1-\beta P)}=\left(-q \beta S+\left(1+q^{2} \beta^{2} P\right)\right) P \\
& =\left(-q \beta(x+y)+1+q^{2} \beta^{2} x y\right) x y=(1-q \beta x)(1-q \beta y) x y .
\end{aligned}
$$

We recall that $\alpha=q^{s}, \beta=1 / a, x=q^{-n-1} / t_{1}, y=q^{-n-1} / t_{2}$, and $s \in$ $\{2, \ldots, n\}$.

From (3.14)-(3.19) we get

$$
\begin{aligned}
= & \frac{\left(q^{-n} / t_{1}, q^{-n} / t_{2} ; q\right)_{s-1} q}{(1-\alpha)(q-\alpha)(q ; q)_{s-2}\left(q^{-2 n-2} /\left(a t_{1} t_{2}\right) ; q\right)_{s+2}\left(1-q^{-2 n} /\left(a t_{1} t_{2}\right)\right)} \\
& \times(1-\alpha)(q-\alpha)(1-\beta x y)(1-q \beta x)(1-q \beta y) x y \\
= & \frac{\left(q^{-n+1} / t_{1}, q^{-n+1} / t_{2} ; q\right)_{s-2}}{\left(q, q^{-2 n+2} /\left(a t_{1} t_{2}\right) ; q\right)_{s-2}} \\
& \times \frac{\left(1-q^{-n} / t_{1}\right)\left(1-q^{-n} / t_{2}\right)\left(1-q^{-n} /\left(a t_{1}\right)\right)\left(1-q^{-n} /\left(a t_{2}\right)\right) q^{-2 n-1} /\left(t_{1} t_{2}\right)}{\left(q^{-2 n-1} /\left(a t_{1} t_{2}\right) ; q\right)_{3}\left(1-q^{-2 n} /\left(a t_{1} t_{2}\right)\right)} \\
= & a^{2} t_{1}^{2} \gamma_{n} q^{2 n-1} B_{s-2}^{(n-1)} .
\end{aligned}
$$

At the end we used formula (3.3) for $\gamma_{n}$.

From (3.12) and (3.20) we obtain

$$
\tilde{r}_{n}(\eta, \mathbf{t})=\gamma_{n} q^{2 n-1} \sum_{j=0}^{n-2} \sum_{k=0}^{j} A_{k} B_{j-k}^{(n-1)} a^{k-j}\left(-1 / t_{1}\right)^{j} \eta^{n-1-j}+K_{n},
$$

where by (3.8), $K_{n}$ is the constant term of

$$
\tilde{r}_{n}(\eta, \mathbf{t})=\left(\eta+\beta_{n} q^{n}\right) \tilde{b}_{n}(\eta, \mathbf{t})-\tilde{b}_{n+1}(\eta, \mathbf{t}) .
$$

We recall that $\tilde{b}_{n}(\eta, \mathbf{t})$ denotes the polynomial on the right-hand side of (3.6). 
To complete the proof it remains to show that $K_{n}$ equals $\gamma_{n} q^{2 n-1}$ times the constant term of $\tilde{b}_{n-1}(\eta, \mathbf{t})$. Let $f_{n}$ denotes the constant term of $\tilde{b}_{n}(\eta, \mathbf{t})$. From (3.22) we have $K_{n}=\beta_{n} q^{n} f_{n}-f_{n+1}$. Therefore, it is enough to verify that

$$
f_{n+1}=\beta_{n} q^{n} f_{n}-\gamma_{n} q^{2 n-1} f_{n-1} .
$$

From (3.6) and (1.15) we have

$$
f_{n}=\frac{\left(q^{-n} / t_{1}, q^{-n} / t_{2} ; q\right)_{n}(-1)^{n}}{\left(q, q^{-2 n} /\left(a t_{1} t_{2}\right) ; q\right)_{n} a^{n} t_{1}^{n}} \phi_{n}=\frac{\left(t_{1} q, t_{2} q ; q\right)_{n} q^{\left(\begin{array}{c}
n \\
2
\end{array}\right)}}{\left(q, a t_{1} t_{2} q^{n+1} ; q\right)_{n} t_{1}^{n}} \phi_{n},
$$

with

$$
\phi_{n}={ }_{4} \phi_{3}\left(\begin{array}{c|c}
q^{-n}, a t_{1} t_{2} q^{n+1}, t_{1}, t_{2} \\
q t_{1}, q t_{2}, a t_{1} t_{2}
\end{array} \mid q, q\right) .
$$

We shall use a recurrence formula for the Askey-Wilson polynomials (see [8] or [12]). The Askey-Wilson polynomials $p_{n}(x ; A, B, C, D \mid q)$ are defined by

$$
p_{n}(x ; A, B, C, D \mid q):={ }_{4} \phi_{3}\left(\begin{array}{c}
q^{-n}, A B C D q^{n-1}, A e^{i \theta}, A e^{-i \theta} \\
A B, A C, A D
\end{array} \mid q, q\right),
$$

where $x=\cos \theta=\left(e^{i \theta}+e^{-i \theta}\right) / 2$. They satisfy the recurrence equation

$$
\begin{gathered}
x p_{n}(x ; A, B, C, D \mid q)=\frac{A_{n}}{2} p_{n+1}(x ; A, B, C, D \mid q) \\
+\frac{B_{n}}{2} p_{n}(x ; A, B, C, D \mid q)+\frac{C_{n}}{2} p_{n-1}(x ; A, B, C, D \mid q), \quad n \geq 0,
\end{gathered}
$$

with $p_{-1}(x ; A, B, C, D \mid q)=0, p_{0}(x ; A, B, C, D \mid q)=1$, and coefficients

$$
\begin{aligned}
& A_{n}=\frac{\left(1-A B q^{n}\right)\left(1-A C q^{n}\right)\left(1-A D q^{n}\right)\left(1-A B C D q^{n-1}\right)}{A\left(1-A B C D q^{2 n-1}\right)\left(1-A B C D q^{2 n}\right)}, \\
& C_{n}=\frac{A\left(1-q^{n}\right)\left(1-B C q^{n-1}\right)\left(1-B D q^{n-1}\right)\left(1-C D q^{n-1}\right)}{\left(1-A B C D q^{2 n-2}\right)\left(1-A B C D q^{2 n-1}\right)} \\
& B_{n}=A+1 / A-A_{n}-C_{n} .
\end{aligned}
$$

As in Section 2 recurrence equation (3.27) can be written in monic form in terms of the polynomials

$$
q_{m}=q_{m}(x ; A, B, C, D \mid q)=p_{m}(x ; A, B, C, D \mid q) 2^{-m} \prod_{j=0}^{m-1} A_{j} .
$$

The monic equation is

$$
x q_{n}=q_{n+1}+\left(B_{n} / 2\right) q_{n}+\left(C_{n} A_{n-1} / 4\right) q_{n-1}, \quad n \geq 0,
$$

$q_{-1}=0, q_{0}=1$. 
We select $A=\sqrt{t_{1} t_{2}}, B=q \sqrt{t_{1} / t_{2}}, C=q \sqrt{t_{2} / t_{1}}, D=a \sqrt{t_{1} t_{2}}$ and $e^{i \theta}=\sqrt{t_{1} / t_{2}}$. Then $x=\cos \theta=\left(t_{1}+t_{2}\right) /\left(2 \sqrt{t_{1} t_{2}}\right)$, and from (3.24)-(3.31) we obtain

$$
\begin{aligned}
& A_{n}=\frac{\left(1-t_{1} q^{n+1}\right)\left(1-t_{2} q^{n+1}\right)\left(1-a t_{1} t_{2} q^{n}\right)\left(1-a t_{1} t_{2} q^{n+1}\right)}{\sqrt{t_{1} t_{2}}\left(1-a t_{1} t_{2} q^{2 n+1}\right)\left(1-a t_{1} t_{2} q^{2 n+2}\right)}, \\
& C_{n}=\frac{\sqrt{t_{1} t_{2}}\left(1-q^{n}\right)\left(1-q^{n+1}\right)\left(1-a t_{1} q^{n}\right)\left(1-a t_{2} q^{n}\right)}{\left(1-a t_{1} t_{2} q^{2 n}\right)\left(1-a t_{1} t_{2} q^{2 n+1}\right)} \\
& B_{n}=\sqrt{t_{1} t_{2}}+1 / \sqrt{t_{1} t_{2}}-A_{n}-C_{n}
\end{aligned}
$$

and

$$
\begin{aligned}
& q_{n}=2^{-n}\left(\prod_{j=0}^{n-1} A_{j}\right) \\
& \times p_{n}\left(\left(t_{1}+t_{2}\right) /\left(2 \sqrt{t_{1} t_{2}}\right) ; \sqrt{t_{1} t_{2}}, q \sqrt{t_{1} / t_{2}}, q \sqrt{t_{2} / t_{1}}, a \sqrt{t_{1} t_{2}} \mid q\right) \\
& =\frac{\left(t_{1} q, t_{2} q, a t_{1} t_{2}, a t_{1} t_{2} q ; q\right)_{n}}{2^{n}\left(t_{1} t_{2}\right)^{n / 2}\left(a t_{1} t_{2} q ; q\right)_{2 n}} \phi_{n}=\frac{\left(t_{1} q, t_{2} q, a t_{1} t_{2} ; q\right)_{n}}{2^{n}\left(t_{1} t_{2}\right)^{n / 2}\left(a t_{1} t_{2} q^{n+1} ; q\right)_{n}} \phi_{n} \\
& =\frac{\left(q, a t_{1} t_{2} ; q\right)_{n}\left(t_{1} / t_{2}\right)^{n / 2}}{2^{n} q^{\left(\begin{array}{c}
n \\
2
\end{array}\right)}} f_{n} .
\end{aligned}
$$

Furthermore, from (3.31) and (3.34) we have

$$
\begin{aligned}
q_{n+1} & =\left(\frac{t_{1}+t_{2}}{2 \sqrt{t_{1} t_{2}}}-\frac{t_{1} t_{2}+1}{2 \sqrt{t_{1} t_{2}}}+\frac{A_{n}}{2}+\frac{C_{n}}{2}\right) q_{n}-\frac{C_{n} A_{n-1}}{4} q_{n-1} \\
& =:\left(R_{n} /\left(2 \sqrt{t_{1} t_{2}}\right)\right) q_{n}-\left(C_{n} A_{n-1} / 4\right) q_{n-1} .
\end{aligned}
$$

From (3.35), (3.36), (3.32), (3.33), and (3.3) we get

$$
\begin{aligned}
f_{n+1}= & \frac{R_{n} q^{n}}{\left(1-q^{n+1}\right)\left(1-a t_{1} t_{2} q^{n}\right) t_{1}} f_{n}-\frac{4 q^{2 n-1}}{\left(q^{n}, a t_{1} t_{2} q^{n-1} ; q\right)_{2} t_{1} / t_{2}} \\
& \times \frac{1}{4} \frac{\left(1-q^{n}\right)\left(1-q^{n+1}\right)\left(1-a t_{1} q^{n}\right)\left(1-a t_{2} q^{n}\right)}{\left(1-a t_{1} t_{2} q^{2 n}\right)\left(1-a t_{1} t_{2} q^{2 n+1}\right)} \\
& \times \frac{\left(1-t_{1} q^{n}\right)\left(1-t_{2} q^{n}\right)\left(1-a t_{1} t_{2} q^{n-1}\right)\left(1-a t_{1} t_{2} q^{n}\right)}{\left(1-a t_{1} t_{2} q^{2 n-1}\right)\left(1-a t_{1} t_{2} q^{2 n}\right)} f_{n-1} \\
= & \frac{R_{n} q^{n}}{\left(1-q^{n+1}\right)\left(1-a t_{1} t_{2} q^{n}\right) t_{1}} f_{n}-\gamma_{n} q^{2 n-1} f_{n-1} .
\end{aligned}
$$

To complete the proof of (3.23) and the theorem we have to show that the coefficient of $f_{n}$ in (3.37) equals $\beta_{n} q^{n}$. From (3.36), (3.32), and (3.33) we 
get

$$
\begin{gathered}
\left(a t_{1} t_{2} q^{2 n} ; q\right)_{3} R_{n}=-\left(1-t_{1}\right)\left(1-t_{2}\right)\left(a t_{1} t_{2} q^{2 n} ; q\right)_{3} \\
+\left(1-t_{1} q^{n+1}\right)\left(1-t_{2} q^{n+1}\right)\left(1-a t_{1} t_{2} q^{n}\right)\left(1-a t_{1} t_{2} q^{n+1}\right)\left(1-a t_{1} t_{2} q^{2 n}\right) \\
+\left(1-q^{n}\right)\left(1-q^{n+1}\right)\left(1-a t_{1} q^{n}\right)\left(1-a t_{2} q^{n}\right)\left(1-a t_{1} t_{2} q^{2 n+2}\right) t_{1} t_{2} .
\end{gathered}
$$

Setting $S=t_{1}+t_{2}, P=t_{1} t_{2}$, and $\alpha=q^{n}$ we obtain

$$
\begin{aligned}
\left(a t_{1} t_{2} q^{2 n} ; q\right)_{3} R_{n}= & -(1-S+P)\left(1-a \alpha^{2} P\right)\left(1-a q \alpha^{2} P\right)\left(1-a q^{2} \alpha^{2} P\right) \\
& +\left(1-q \alpha S+q^{2} \alpha^{2} P\right)(1-a \alpha P)(1-a q \alpha P)\left(1-a \alpha^{2} P\right) \\
& +(1-\alpha)(1-q \alpha)\left(1-a \alpha S+a^{2} \alpha^{2} P\right)\left(1-a q^{2} \alpha^{2} P\right) P \\
= & : E_{1} S+E_{2} P+E_{3} P .
\end{aligned}
$$

The expressions $\left\{E_{j}\right\}_{j=1}^{3}$ are defined and factored below:

$$
\begin{aligned}
E_{1}:= & \left(1-a \alpha^{2} P\right)\left(1-a q \alpha^{2} P\right)\left(1-a q^{2} \alpha^{2} P\right) \\
& -q \alpha(1-a \alpha P)(1-a q \alpha P)\left(1-a \alpha^{2} P\right) \\
& -a \alpha(1-\alpha)(1-q \alpha)\left(1-a q^{2} \alpha^{2} P\right) P \\
= & \left(1-a \alpha^{2} P\right)\left[1-a q \alpha^{2} P-a q^{2} \alpha^{2} P+a^{2} q^{3} \alpha^{4} P^{2}\right. \\
& \left.-q \alpha+a q \alpha^{2} P+a q^{2} \alpha^{2} P-a^{2} q^{2} \alpha^{3} P^{2}\right] \\
& -a \alpha(1-\alpha)(1-q \alpha)\left(1-a q^{2} \alpha^{2} P\right) P \\
= & (1-q \alpha)\left(\left(1-a \alpha^{2} P\right)\left(1-a^{2} q^{2} \alpha^{3} P^{2}\right)\right. \\
& \left.-a \alpha(1-\alpha)\left(1-a q^{2} \alpha^{2} P\right) P\right) \\
= & (1-q \alpha)\left(1+a^{3} q^{2} \alpha^{5} P^{3}-a \alpha P-a^{2} q^{2} \alpha^{4} P^{2}\right) \\
= & (1-q \alpha)(1-a \alpha P)\left(1-a^{2} q^{2} \alpha^{4} P^{2}\right),
\end{aligned}
$$

(3.40)

$$
\begin{aligned}
E_{2}:= & \left(-\left(1-a \alpha^{2} P\right)\left(1-a q \alpha^{2} P\right)\left(1-a q^{2} \alpha^{2} P\right)\right. \\
& \left.+(1-a \alpha P)(1-a q \alpha P)\left(1-a \alpha^{2} P\right)\right) / P \\
= & \left(1-a \alpha^{2} P\right) a \alpha\left(\alpha q+\alpha q^{2}-a q^{3} \alpha^{3} P-1-q+a q \alpha P\right) \\
= & a \alpha(1-q \alpha)\left(1-a \alpha^{2} P\right)(-(1+q)+(1+q \alpha) a q \alpha P) \\
= & -a \alpha(1-q \alpha)\left[1+q-a \alpha((1+q) \alpha+q(1+q \alpha)) P+a^{2} q \alpha^{3}(1+q \alpha) P^{2}\right],
\end{aligned}
$$


and

$$
\begin{aligned}
E_{3}:= & -\left(1-a \alpha^{2} P\right)\left(1-a q \alpha^{2} P\right)\left(1-a q^{2} \alpha^{2} P\right) \\
& +q^{2} \alpha^{2}(1-a \alpha P)(1-a q \alpha P)\left(1-a \alpha^{2} P\right) \\
& +(1-\alpha)(1-q \alpha)\left(1+a^{2} \alpha^{2} P\right)\left(1-a q^{2} \alpha^{2} P\right) \\
= & \left(1-a \alpha^{2} P\right)\left(-1+a q(1+q) \alpha^{2} P+q^{2} \alpha^{2}-a q^{2}(1+q) \alpha^{3} P\right) \\
& +(1-\alpha)(1-q \alpha)\left(1+a\left(a-q^{2}\right) \alpha^{2} P-a^{3} q^{2} \alpha^{4} P^{2}\right) \\
= & (1-q \alpha)\left(1-a \alpha^{2} P\right)\left(-1-q \alpha+a q(1+q) \alpha^{2} P\right) \\
& +(1-q \alpha)(1-\alpha)\left(1+a\left(a-q^{2}\right) \alpha^{2} P-a^{3} q^{2} \alpha^{4} P^{2}\right) \\
= & (1-q \alpha)\left[-(1+q) \alpha+a \alpha^{2}\left(q(1+q)+1+q \alpha+(1-\alpha)\left(a-q^{2}\right)\right) P\right. \\
& \left.-a^{2} q \alpha^{4}(1+q+a q(1-\alpha)) P^{2}\right] .
\end{aligned}
$$

Combining (3.40) and (3.41) we obtain

$$
\begin{aligned}
\left(E_{2}+E_{3}\right) /(1-q \alpha)= & -(1+q)(1+a) \alpha \\
& +a \alpha^{2}\left(a \alpha+a q \alpha+a q+a q^{2} \alpha+q\right. \\
& \left.+1+q \alpha+a-a \alpha+q^{2} \alpha\right) P \\
& -a^{2} q \alpha^{4}(a(1+q \alpha)+1+q+a q(1-\alpha)) P^{2} \\
= & -(1+q)(1+a) \alpha+a \alpha^{2}(1+q)(a q \alpha+a+1+q \alpha) P \\
& -a^{2} q \alpha^{4}(1+q)(1+a) P^{2} \\
= & -(1+q)(1+a) \alpha\left(1-a \alpha(1+q \alpha) P+a^{2} q \alpha^{3} P^{2}\right) \\
= & -(1+q)(1+a) \alpha(1-a \alpha P)\left(1-a q \alpha^{2} P\right) .
\end{aligned}
$$

Then from (3.38), (3.39), and (3.42) we get

$$
\begin{aligned}
\left(a t_{1} t_{2} q^{2 n} ; q\right){ }_{3} R_{n}= & (1-q \alpha)(1-a \alpha P)\left(1-a q \alpha^{2} P\right) \\
& \times\left(\left(1+a q \alpha^{2} P\right) S-(1+q)(1+a) \alpha P\right) .
\end{aligned}
$$

In terms of $\alpha=q^{n}, S=t_{1}+t_{2}$, and $P=t_{1} t_{2},\left(a t_{1} t_{2} q^{2 n} ; q\right)_{3}=\left(a \alpha^{2} P ; q\right)_{3}$, hence (3.43) implies

$$
\begin{gathered}
\frac{R_{n}}{\left(1-q^{n+1}\right)\left(1-a t_{1} t_{2} q^{n}\right) t_{1}}=\frac{R_{n}}{(1-q \alpha)(1-a \alpha P) t_{1}} \\
=\frac{\left(1+a q \alpha^{2} P\right) S-(1+q)(1+a) \alpha P}{\left(1-a \alpha^{2} P\right)\left(1-a q^{2} \alpha^{2} P\right) t_{1}}=\beta_{n}
\end{gathered}
$$

in view of (3.4). The proof of Theorem 3.1 is complete. 


\section{The Spectrum of the Inverse Operator.}

To find the spectrum of the operator $\mathcal{T}_{\mathbf{t}}$, that is, to solve eigenvalue problem (2.4) we consider the eigenfunctions of the $q$-difference operator $D_{q, x}$. It is easy to see that for every $\lambda$ the equation $D_{q, x} f(x)=\lambda f(x)$ has solution $f_{\lambda}(x)=1 /(\lambda(1-q) x ; q)_{\infty}=e_{q}(\lambda(1-q) x)$. The eigenfunctions of $\mathcal{T}_{\mathbf{t}}$ are also eigenfunctions for $D_{q, x}$, in fact if $g_{\lambda}$ is such that $\mathcal{T}_{\mathbf{t}} g_{\lambda}=\lambda g_{\lambda}$, then $g_{\lambda}=D_{q, x} \mathcal{T}_{\mathbf{t}} g_{\lambda}=\lambda D_{q, x} g_{\lambda}$ and therefore, $g_{\lambda}(x)=f_{1 / \lambda}(x)=e_{q}((1-q) x / \lambda)$. Hence, the eigenvalues of $\mathcal{T}_{\mathbf{t}}$ are the reciprocals of the numbers $\lambda$ such that

$$
f_{\lambda}(x)=\sum_{n=0}^{\infty} c_{n}(\lambda, \mathbf{t}) p_{n}(x, \mathbf{t}) \in L_{2}(\mu(\cdot, \mathbf{t})) \cap L_{2}(\mu(\cdot, q \mathbf{t})) .
$$

From equations $\mathcal{T}_{\mathbf{t}} g_{\lambda}=\lambda g_{\lambda}, g_{\lambda}=f_{1 / \lambda},(2.2)$, and (4.1) we get $c_{0}(1 / \lambda, \mathbf{t})=0$. It turns out that the condition $c_{0}(1 / \lambda, \mathbf{t})=0$ completely characterizes the spectrum of the operator $\mathcal{T}_{\mathbf{t}}$.

We proceed with computing the coefficients $\left\{c_{n}(\lambda, \mathbf{t})\right\}$ in (4.1). Let $\lambda$ be small enough so that $|\lambda(1-q)|<1$. Using the orthogonality relation (1.6) and applying Euler's identity $(1.12)$ to $f_{\lambda}(x)=e_{q}(\lambda(1-q) x)$ we obtain

$$
c_{n}(\lambda, \mathbf{t}) \xi_{n}(\mathbf{t})=\int_{\mathbf{R}} f_{\lambda}(x) p_{n}(x, \mathbf{t}) d \mu(x, \mathbf{t})=\sum_{s=0}^{\infty} \frac{(\lambda(1-q))^{s}}{(q ; q)_{s}} d_{n, s}(\lambda, \mathbf{t}),
$$

where we have defined

$$
d_{n, s}(\lambda, \mathbf{t}):=\int_{\mathbf{R}} x^{s} p_{n}(x, \mathbf{t}) d \mu(x, \mathbf{t}) .
$$

By (1.6), $d_{n, s}=0$ if $s<n$, hence we may assume that $s \geq n$.

From (1.11) and (1.3) we have

$$
\begin{aligned}
& x^{s}=\frac{1}{t_{2}^{s}} \sum_{j=0}^{s} a_{s, j}\left(t_{2} x ; q\right)_{j}, \quad a_{s, j}:=(-1)^{j} q^{\left(\begin{array}{c}
j \\
2
\end{array}\right)-j(s-1)}\left[\begin{array}{l}
s \\
j
\end{array}\right]_{q}, \\
& p_{n}(x, \mathbf{t})=\sum_{k=0}^{n} b_{n, k}\left(t_{1} x ; q\right)_{k}, \quad b_{n, k}:=\frac{\left(q^{-n}, a t_{1} t_{2} q^{n-1} ; q\right)_{k}}{\left(t_{1}, a t_{1}, q ; q\right)_{k}} q^{k} .
\end{aligned}
$$

Then for $d_{n, s}(\lambda, \mathbf{t})$ we get

$$
\begin{aligned}
d_{n, s}(\lambda, \mathbf{t}) & =\sum_{j=0}^{s} \sum_{k=0}^{n} a_{s, j} b_{n, k} \frac{1}{t_{2}^{s}} \int_{\mathbf{R}} \frac{d \mu^{(a)}(x)}{\left(q^{k} t_{1} x, q^{j} t_{2} x ; q\right)_{\infty}} \\
& =\sum_{j=0}^{s} \sum_{k=0}^{n} a_{s, j} b_{n, k} \frac{1}{t_{2}^{s}} \frac{\left(a t_{1} t_{2} q^{k+j} ; q\right)_{\infty}}{\left(q^{k} t_{1}, q^{j} t_{2}, a q^{k} t_{1}, a q^{j} t_{2} ; q\right)_{\infty}},
\end{aligned}
$$


where (6.4) was used to evaluate the integrals. From the formulas for $a_{s, j}$ and $b_{n, k}$, and (4.4) we obtain

$$
\begin{aligned}
d_{n, s}= & \frac{\left(a t_{1} t_{2} ; q\right)_{\infty}(q ; q)_{s}}{\left(t_{1}, a t_{1}, t_{2}, a t_{2} ; q\right)_{\infty} t_{2}^{s}} \\
& \times \sum_{j=0}^{s} \sum_{k=0}^{n} \frac{(-1)^{j} q^{\left(\begin{array}{c}
j \\
2
\end{array}\right)-j(s-1)+k}\left(q^{-n}, a t_{1} t_{2} q^{n-1} ; q\right)_{k}\left(t_{2}, a t_{2} ; q\right)_{j}}{(q ; q)_{j}(q ; q)_{s-j}(q ; q)_{k}\left(a t_{1} t_{2} ; q\right)_{k+j}} \\
= & c^{(1)} \sum_{j=0}^{s} \frac{(-1)^{j} q^{\left(\begin{array}{c}
j \\
2
\end{array}\right)-j(s-1)}\left(t_{2}, a t_{2} ; q\right)_{j}}{(q ; q)_{j}(q ; q)_{s-j}\left(a t_{1} t_{2} ; q\right)_{j}}{ }^{2} \phi_{1}\left(\begin{array}{c}
q^{-n}, a t_{1} t_{2} q^{n-1} \\
a t_{1} t_{2} q^{j}
\end{array} \mid q, q\right),
\end{aligned}
$$

where $c^{(1)}$ denotes the coefficient of the double sum. By (1.9) the ${ }_{2} \phi_{1}$ sum is equal to

$$
\frac{\left(q^{j+1-n} ; q\right)_{n}\left(a t_{1} t_{2} q^{n-1}\right)^{n}}{\left(a t_{1} t_{2} q^{j} ; q\right)_{n}},
$$

which is 0 for $j<n$. Then for $s \geq n$ we obtain

$$
d_{n, s}=c^{(2)} \sum_{j=n}^{s} \frac{(-1)^{j} q^{\left(\begin{array}{l}
j \\
2
\end{array}\right)-j(s-1)}\left(t_{2}, a t_{2} ; q\right)_{j}}{(q ; q)_{j-n}(q ; q)_{s-j}\left(a t_{1} t_{2} ; q\right)_{n+j}}
$$

where $c^{(2)}=\left(a t_{1} t_{2} q^{n-1}\right)^{n} c^{(1)}$ and we used the identity $\left(q^{j+1-n} ; q\right)_{n}=(q ; q)_{j} /$ $(q ; q)_{j-n}$. Replacing $j$ by $n+l$ we get

$$
d_{n, s}=c^{(3)} \sum_{l=0}^{s-n} \frac{(-1)^{l} q^{\left(\begin{array}{c}
n+l \\
2
\end{array}\right)-(n+l)(s-1)}\left(t_{2} q^{n}, a t_{2} q^{n} ; q\right)_{l}}{(q ; q)_{l}(q ; q)_{(s-n)-l}\left(a t_{1} t_{2} q^{2 n} ; q\right)_{l}}, \quad s \geq n,
$$

with $c^{(3)}=(-1)^{n}\left(t_{2}, a t_{2} ; q\right)_{n} /\left(a t_{1} t_{2} ; q\right)_{2 n} c^{(2)}$. Next with $p=s-n$ we have

$$
\frac{(q ; q)_{p}}{(q ; q)_{p-l}}=\prod_{j=p-l+1}^{p}\left(1-q^{j}\right)=(-1)^{l} q^{\left(\begin{array}{c}
p+1 \\
2
\end{array}\right)-\left(\begin{array}{c}
p-l+1 \\
2
\end{array}\right)}\left(q^{-p} ; q\right)_{l}
$$

and

$$
\left(\begin{array}{c}
n+l \\
2
\end{array}\right)-(n+l)(s-1)+\left(\begin{array}{c}
p+1 \\
2
\end{array}\right)-\left(\begin{array}{c}
p-l+1 \\
2
\end{array}\right)=\left(\begin{array}{l}
n \\
2
\end{array}\right)-n(s-1)+l .
$$

Substituting these identities in (4.7) we obtain

$$
d_{n, s}=c^{(4)}{ }_{3} \phi_{2}\left(\begin{array}{c}
q^{-(s-n)}, t_{2} q^{n}, a t_{2} q^{n} \\
a t_{1} t_{2} q^{2 n}, 0
\end{array} \mid q, q\right), \quad s \geq n,
$$

where

$$
c^{(4)}=c_{n, s}^{(4)}=\frac{\left(a t_{1} t_{2} q^{2 n} ; q\right)_{\infty}\left(t_{2}, a t_{2} ; q\right)_{n}\left(a t_{1} t_{2}\right)^{n}(q ; q)_{s} q^{\left(\begin{array}{c}
n \\
2
\end{array}\right)+n(n-s)}(-1)^{n}}{\left(t_{1}, a t_{1}, t_{2}, a t_{2} ; q\right)_{\infty}(q ; q)_{s-n} t_{2}^{s}} .
$$


From formula (1.7) for $\xi_{n}(\mathbf{t})$ and (4.9) we get

$$
c_{n, s}^{(4)}=\frac{\left(t_{1}, a t_{1} ; q\right)_{n}(q ; q)_{s} q^{n(n-s)} t_{2}^{n-s}}{\left(a t_{1} t_{2} q^{n-1}, q ; q\right)_{n}(q ; q)_{s-n} t_{1}^{n}} \xi_{n}(\mathbf{t}) .
$$

Then from (4.2), (4.8), (4.9), and (4.10) we get

$$
\begin{aligned}
& c_{n}(\lambda, \mathbf{t})=\sum_{s=n}^{\infty} \frac{((1-q) \lambda)^{s}}{(q ; q)_{s}}\left(d_{n, s} / \xi_{n}(\mathbf{t})\right)=\frac{\left(t_{1}, a t_{1} ; q\right)_{n}(1-q)^{n}}{\left(a t_{1} t_{2} q^{n-1}, q ; q\right)_{n} t_{1}^{n}} \lambda^{n} \\
& \times \sum_{k=0}^{\infty} \frac{\left((1-q) /\left(t_{2} q^{n}\right)\right)^{k}}{(q ; q)_{k}} \lambda^{k}{ }_{3} \phi_{2}\left(\begin{array}{c}
q^{-k}, t_{2} q^{n}, a t_{2} q^{n} \\
a t_{1} t_{2} q^{2 n}, 0
\end{array} \mid q, q\right),
\end{aligned}
$$

with $k=s-n$. Applying the transformation, [6],

$$
{ }_{3} \phi_{2}\left(\begin{array}{c}
q^{-k}, a, b \\
c, 0
\end{array} \mid q, q\right)=\frac{(b ; q)_{k} a^{k}}{(c ; q)_{k}}{ }_{2} \phi_{1}\left(\begin{array}{c}
q^{-k}, c / b \\
q^{1-k} / b
\end{array} \mid q, q / a\right)
$$

to the ${ }_{3} \phi_{2}$ expression in (4.11) we get

$$
\begin{aligned}
& { }_{3} \phi_{2}\left(\begin{array}{c}
q^{-k}, t_{2} q^{n}, a t_{2} q^{n} \\
a t_{1} t_{2} q^{2 n}, 0
\end{array} \mid q, q\right) \\
& =\frac{\left(a t_{2} q^{n} ; q\right)_{k}\left(t_{2} q^{n}\right)^{k}}{\left(a t_{1} t_{2} q^{2 n} ; q\right)_{k}} 2 \phi_{1}\left(\begin{array}{c}
q^{-k}, t_{1} q^{n} \\
q^{1-k-n} /\left(a t_{2}\right)
\end{array} \mid q, q^{1-n} / t_{2}\right) .
\end{aligned}
$$

Then the second sum in (4.11) can be written in the form

$$
\sum_{k=0}^{\infty} \frac{\left(a t_{2} q^{n} ; q\right)_{k}(1-q)^{k}}{\left(a t_{1} t_{2} q^{2 n}, q ; q\right)_{k}} \lambda^{k} \sum_{j=0}^{k} \frac{\left(q^{-k} ; q\right)_{j}\left(t_{1} q^{n} ; q\right)_{j}\left(q^{1-n} / t_{2}\right)^{j}}{\left(q^{1-k-n} /\left(a t_{2}\right) ; q\right)_{j}(q ; q)_{j}}
$$

Using (1.15) we obtain

$$
\frac{\left(q^{-k} ; q\right)_{j}}{\left(q^{1-k-n} /\left(a t_{2}\right) ; q\right)_{j}}=\frac{(q ; q)_{k}\left(a t_{2} q^{n} ; q\right)_{k-j}}{(q ; q)_{k-j}\left(a t_{2} q^{n} ; q\right)_{k}}\left(a t_{2} q^{n-1}\right)^{j} .
$$

Hence the double sum in (4.13) equals

$$
\sum_{k=0}^{\infty} \frac{((1-q) \lambda)^{k}}{\left(a t_{1} t_{2} q^{2 n}, q ; q\right)_{k}} \sum_{j=0}^{k}\left[\begin{array}{l}
k \\
j
\end{array}\right]_{q}\left(t_{1} q^{n} ; q\right)_{j}\left(a t_{2} q^{n} ; q\right)_{k-j} a^{j} .
$$

The above formulas hold when $|(1-q) \lambda|<1$ since in this range (1.12) can be applied. To extend the formulas to arbitrary $\lambda$ we need a meromorphic continuation of the function in (4.14). We set

$$
\begin{aligned}
\alpha_{k}\left(a, t_{1}, t_{2}\right) & :=\frac{1}{\left(a t_{1} t_{2}, q ; q\right)_{k}} \sum_{j=0}^{k}\left[\begin{array}{c}
k \\
j
\end{array}\right]_{q}\left(t_{1} ; q\right)_{j}\left(a t_{2} ; q\right)_{k-j} a^{j}, \\
A\left(z ; a, t_{1}, t_{2}\right) & :=\sum_{k=0}^{\infty} \alpha_{k}\left(a, t_{1}, t_{2}\right) z^{k}, \quad|z|<1 .
\end{aligned}
$$


Note that the sum in (4.14) equals $A\left((1-q) \lambda ; a, q^{n} \mathbf{t}\right)$. For $|z|<1$ we consider the product of the functions $(z, a z ; q)_{\infty}$ and $A\left(z ; a, t_{1}, t_{2}\right)$. Using (1.13) we get

$$
(z ; q)_{\infty} A\left(z ; a, t_{1}, t_{2}\right)=\sum_{n=0}^{\infty} z^{n}\left(\sum_{k=0}^{n} \alpha_{k}\left(a, t_{1}, t_{2}\right) \frac{(-1)^{n-k} q^{\left(\begin{array}{c}
n-k \\
2
\end{array}\right)}}{(q ; q)_{n-k}}\right) .
$$

The coefficient of $z^{n}$ in (4.17) equals

$$
\begin{aligned}
& \sum_{k=0}^{n}\left(\sum_{j=0}^{k}\left[\begin{array}{c}
k \\
j
\end{array}\right]_{q} \frac{\left(t_{1} ; q\right)_{j}\left(a t_{2} ; q\right)_{k-j} a^{j}}{\left(a t_{1} t_{2}, q ; q\right)_{k}}\right) \frac{(-1)^{n-k} q^{\left(\begin{array}{c}
n-k \\
2
\end{array}\right)}}{(q ; q)_{n-k}} \\
& =\sum_{j=0}^{n} \frac{\left(t_{1} ; q\right)_{j} a^{j}}{\left(a t_{1} t_{2}, q ; q\right)_{j}(q ; q)_{n-j}}\left(\sum_{\nu=0}^{n-j}\left[\begin{array}{c}
n-j \\
\nu
\end{array}\right]_{q} \frac{\left(a t_{2} ; q\right)_{\nu}(-1)^{n-j-\nu} q^{\left(\begin{array}{c}
n-j-\nu \\
2
\end{array}\right)}}{\left(a t_{1} t_{2} q^{j} ; q\right)_{\nu}}\right)
\end{aligned}
$$

with $\nu=k-j$. The sum over $\nu$ in (4.18) has the form

$$
\begin{aligned}
\sum_{\nu=0}^{m}\left[\begin{array}{c}
m \\
\nu
\end{array}\right]_{q} & \frac{(\alpha ; q)_{\nu}}{(\beta ; q)_{\nu}}(-1)^{m-\nu} q^{\left(\begin{array}{c}
m-\nu \\
2
\end{array}\right)} \\
= & (-1)^{m} q^{\left(\begin{array}{c}
m \\
2
\end{array}\right)} \sum_{\nu=0}^{m} \frac{\left(q^{-m}, \alpha ; q\right)_{\nu}}{(\beta, q ; q)_{\nu}} q^{\nu} \\
= & (-1)^{m} q^{\left(\begin{array}{c}
m \\
2
\end{array}\right)}{ }_{2} \phi_{1}\left(\begin{array}{c}
q^{-m} \\
\beta
\end{array} \mid q, q\right) \\
= & (-1)^{m} q^{\left(\begin{array}{c}
m \\
2
\end{array}\right)} \frac{(\beta / \alpha ; q)_{m}}{(\beta ; q)_{m}} \alpha^{m},
\end{aligned}
$$

where we first used the identity

$$
(q ; q)_{m} /(q ; q)_{m-\nu}=(-1)^{\nu} q^{\left(\begin{array}{c}
m+1 \\
2
\end{array}\right)-\left(\begin{array}{c}
m-\nu+1 \\
2
\end{array}\right)}\left(q^{-m} ; q\right)_{\nu}
$$

and then (1.9). Then the coefficient of $z^{n}$ in (4.17) given with (4.18) equals

$$
\begin{aligned}
& \sum_{j=0}^{n} \frac{\left(t_{1} ; q\right)_{j} a^{j}}{\left(a t_{1} t_{2}, q ; q\right)_{j}(q ; q)_{n-j}}(-1)^{n-j} q^{\left(\frac{n-j}{2}\right)} \frac{\left(t_{1} q^{j} ; q\right)_{n-j}\left(a t_{2}\right)^{n-j}}{\left(a t_{1} t_{2} q^{j} ; q\right)_{n-j}} \\
= & \frac{\left(t_{1} ; q\right)_{n} a^{n}}{\left(a t_{1} t_{2}, q ; q\right)_{n}} \sum_{j=0}^{n}\left[\begin{array}{c}
n \\
n-j
\end{array}\right]_{q} q^{\left(\frac{n-j}{2}\right)}\left(-t_{2}\right)^{n-j}=\frac{\left(t_{1}, t_{2} ; q\right)_{n} a^{n}}{\left(a t_{1} t_{2}, q ; q\right)_{n}},
\end{aligned}
$$

where we applied (1.14). From (4.17) and (4.20) we get

$$
(z ; q)_{\infty} A\left(z ; a, t_{1}, t_{2}\right)={ }_{2} \phi_{1}\left(\begin{array}{c}
t_{1}, t_{2} \\
a t_{1} t_{2}
\end{array} \mid q, a z\right), \quad|a z|<1 .
$$


From (1.13) and (4.21) for $z$ such that $\max \{|z|,|a z|\}<1$ we have

$$
\begin{aligned}
& (z, a z ; q)_{\infty} A\left(z ; a, t_{1}, t_{2}\right) \\
& =\sum_{m=0}^{\infty}\left(\sum_{k=0}^{m} \frac{\left(t_{1}, t_{2} ; q\right)_{k}}{\left(a t_{1} t_{2}, q ; q\right)_{k}} \frac{(-1)^{m-k} q^{\left(\begin{array}{c}
m-k \\
2
\end{array}\right)}}{(q ; q)_{m-k}}\right) a^{m} z^{m} \\
& =\sum_{m=0}^{\infty} \frac{(-1)^{m} q^{\left(\begin{array}{c}
m \\
2
\end{array}\right)}}{(q ; q)_{m}}{ }_{3} \phi_{2}\left(\begin{array}{c}
q^{-m}, t_{1}, t_{2} \\
a t_{1} t_{2}, 0
\end{array} \mid q, q\right) a^{m} z^{m} \\
& =\sum_{m=0}^{\infty} \frac{\left(-t_{1}\right)^{m} q^{\left(\begin{array}{c}
m \\
2
\end{array}\right)}\left(t_{2} ; q\right)_{m}}{\left(a t_{1} t_{2}, q ; q\right)_{m}}{ }_{2} \phi_{1}\left(\begin{array}{c}
q^{-m}, a t_{1} \\
q^{1-m} / t_{2}
\end{array} \mid q, q / t_{1}\right) a^{m} z^{m},
\end{aligned}
$$

where we applied (4.19) and (4.12). Next, from (1.15) we have

$$
\frac{\left(q^{-m} ; q\right)_{j}}{\left(q^{1-m} / t_{2} ; q\right)_{j}}=\frac{(q ; q)_{m}\left(t_{2} ; q\right)_{m-j}}{(q ; q)_{m-j}\left(t_{2} ; q\right)_{m}}\left(t_{2} / q\right)^{j},
$$

which combined with (4.22) yields

$$
\begin{aligned}
(z, a z ; q)_{\infty} A\left(z ; a, t_{1}, t_{2}\right)= & \sum_{m=0}^{\infty} \frac{\left(-t_{1}\right)^{m} q^{\left(\begin{array}{c}
m \\
2
\end{array}\right)}}{\left(a t_{1} t_{2} ; q\right)_{m}} \\
& \times\left(\sum_{j=0}^{m} \frac{\left(a t_{1} ; q\right)_{j}\left(t_{2} ; q\right)_{m-j}}{(q ; q)_{j}(q ; q)_{m-j}}\left(t_{2} / t_{1}\right)^{j}\right) a^{m} z^{m} .
\end{aligned}
$$

Clearly $(z, a z ; q)_{\infty}$ is an entire function of $z$. Furthermore, the right-hand side of (4.23) is an entire function of $z$, and in an open neighborhood of $z=0$ it coincides with the function $(z, a z ; q)_{\infty} A\left(z ; a, t_{1}, t_{2}\right)$. Hence a meromorphic extension of $A\left(z ; a, t_{1}, t_{2}\right)$ to the complex plane can be found by dividing the right-hand side of $(4.23)$ by $(z, a z ; q)_{\infty}$.

The main results of this section can be described with the following two theorems.

Theorem 4.1. The coefficients in the expansion formula for the eigenfunction $f_{\lambda}(x)=e_{q}(\lambda(1-q) x)$ in terms of big q-Jacobi polynomials $\left\{p_{n}(x, \mathbf{t})\right\}$ are given by

$$
\begin{gathered}
c_{n}(\lambda, \mathbf{t})=\frac{1}{((1-q) \lambda, a(1-q) \lambda ; q)_{\infty}} \frac{\left(t_{1}, a t_{1} ; q\right)_{n}(1-q)^{n}}{\left(a t_{1} t_{2} q^{n-1}, q ; q\right)_{n} t_{1}^{n}} \lambda^{n} \\
\times \sum_{m=0}^{\infty} \frac{\left(-t_{1} q^{n}\right)^{m} q^{\left(\begin{array}{c}
m \\
2
\end{array}\right)}}{\left(a t_{1} t_{2} q^{2 n} ; q\right)_{m}}\left(\sum_{j=0}^{m} \frac{\left(a t_{1} q^{n} ; q\right)_{j}\left(t_{2} q^{n} ; q\right)_{m-j}}{(q ; q)_{j}(q ; q)_{m-j}}\left(\frac{t_{2}}{t_{1}}\right)^{j}\right) a^{m}(1-q)^{m} \lambda^{m} .
\end{gathered}
$$


Furthermore, the coefficients $\left\{c_{n}(\lambda, \mathbf{t})\right\}$ satisfy the recurrence equation $(4.25)$

$$
\sigma_{n}(\mathbf{t}) c_{n}(\lambda, \mathbf{t})=\lambda \sum_{m=n-1}^{n+1}\left(\xi_{m}(\mathbf{t}) / \xi_{n-1}(q \mathbf{t})\right) c_{n-1, m}(\mathbf{t}) c_{m}(\lambda, \mathbf{t}), \quad n \geq 1,
$$

where $\left\{c_{n, m}(\mathbf{t})\right\}_{m=n}^{n+2}$ are the coefficients defined with (2.7)-(2.10).

The spectrum of the inverse operator $\mathcal{T}_{\mathbf{t}}$ is described in Theorem 4.2.

Theorem 4.2. The function $f_{\lambda}(x)$ belongs to the space $L_{2}(\mu(\cdot, \mathbf{t}))$ for every $\lambda$ that is not a zero of $((1-q) \lambda, a(1-q) \lambda ; q)_{\infty}$.

The spectrum of $\mathcal{T}_{\mathbf{t}}$, the inverse operator of the q-difference operator $D_{q, x}$, acting on the space $L_{2}(\mu(\cdot, \mathbf{t})) \cap L_{2}(\mu(\cdot, q \mathbf{t}))$ is the set of the reciprocals of the zeros of the meromorphic function $c_{0}(\lambda, \mathbf{t})$.

Proof. From (1.6), (1.7), (4.1), and (4.24) it immediately follows that

$$
\left\|f_{\lambda}\right\|_{L_{2}(\mu(\cdot, \mathbf{t}))}^{2}=\sum_{n=0}^{\infty} c_{n}(\lambda, \mathbf{t})^{2} \xi_{n}(\mathbf{t})<\infty
$$

for all $\lambda$ such that $1 /((1-q) \lambda) \notin \operatorname{supp}\left(\mu^{(a)}\right)$ and all parameters $\mathbf{t}$ for which the function $c_{0}(\lambda, \mathbf{t})$ is well-defined. This is due to the presence of the factor $q^{\left(\begin{array}{l}n \\ 2\end{array}\right)}$ in $\xi_{n}(\mathbf{t})$. Furthermore, $\mathcal{T}_{\mathbf{t}} g_{\lambda}=\lambda g_{\lambda}$ implies $g_{\lambda}=f_{1 / \lambda}$ and $c_{0}(1 / \lambda, \mathbf{t})=0$. Hence $c_{0}(1 / \lambda, \mathbf{t})=0$ is a necessary and sufficient condition for $\lambda$ to be in the spectrum of the operator $\mathcal{T}_{\mathbf{t}}$.

\section{Asymptotic Properties of the Polynomials $\left\{b_{n}(\eta, \mathbf{t})\right\}$.}

In Section 2 we applied Schwartz's theorem to prove that the sequence $\left\{\eta^{n} b_{n}(1 / \eta, \mathbf{t})\right\}$ converges locally uniformly in the complex plane to an entire function. The recurrence relation (2.17) has bounded coefficients, hence the polynomials $\left\{b_{n}(\eta, \mathbf{t})\right\}$ are orthogonal with respect to a unique measure $\varphi(\cdot, \mathbf{t})$ with compact support, [1], [14]. From Markov's theorem, [15], the Stieltjes transform of $\varphi(\cdot, \mathbf{t})$ is given by

$$
\int_{\mathbf{R}} \frac{d \varphi(u, \mathbf{t})}{z-u}=\lim _{n \rightarrow \infty} \frac{b_{n}^{*}(z, \mathbf{t})}{b_{n}(z, \mathbf{t})}, \quad z \notin \mathbf{R},
$$

where $\left\{b_{n}^{*}(\eta, \mathbf{t})\right\}$ is the solution of (2.17) or equivalently, (3.1) satisfying the initial conditions

$$
b_{0}^{*}(\eta, \mathbf{t})=0, \quad b_{1}^{*}(\eta, \mathbf{t})=1 .
$$

We observe that $\beta_{n}(\mathbf{t})$ and $\gamma_{n}(\mathbf{t})$ defined with (3.4) and (3.3) have the property

$$
\beta_{n-1}(q \mathbf{t})=\beta_{n}(\mathbf{t}) \quad \text { and } \quad \gamma_{n-1}(q \mathbf{t})=\gamma_{n}(\mathbf{t}) .
$$


From (3.1) with $n, \mathbf{t}$ and $\eta$ replaced by $n-1, q \mathbf{t}$ and $\eta / q$, respectively, we get

$$
\begin{aligned}
& b_{n}(\eta / q, q \mathbf{t})=\left(\eta / q+\beta_{n-1}(q \mathbf{t}) q^{n-1}\right) b_{n-1}(\eta / q, q \mathbf{t}) \\
& \quad-\gamma_{n-1}(q \mathbf{t}) q^{2 n-3} b_{n-2}(\eta / q, q \mathbf{t}) .
\end{aligned}
$$

Multiplying (5.3) by $q^{n}$ and using (5.2) we see that

$$
b_{n}^{*}(\eta, \mathbf{t})=q^{n-1} b_{n-1}(\eta / q, q \mathbf{t}), \quad n \geq 0 .
$$

We now study the limiting behavior of $\eta^{n} b_{n}(1 / \eta, \mathbf{t})$ as $n \rightarrow \infty$. For each fixed $j$ the ${ }_{4} \phi_{3}$ expression in (3.6) is bounded by $M_{1}(\mathbf{t})_{1} \phi_{0}\left(q^{-j} ;-; q,-q\right)$ and the coefficient of the ${ }_{4} \phi_{3}$ is bounded by $M_{2}(\mathbf{t})\left|\eta / t_{1}\right|{ }^{j} q^{j(j-1) / 2} /(q ; q)_{j}$. Here both $M_{1}(\mathbf{t})$ and $M_{2}(\mathbf{t})$ are positive and depend only on $\mathbf{t}$. The following estimate

$$
\sum_{j=0}^{\infty}\left|\frac{\eta}{t_{1}}\right|^{j} \frac{q^{\left(\begin{array}{l}
j \\
2
\end{array}\right)}}{(q ; q)_{j}}{ }_{2} \phi_{1}\left(q^{-j} ;-; q,-q\right) \leq \sum_{j=0}^{\infty} \frac{\left|\eta / t_{1}\right|^{j}}{(q ; q)_{j}} \sum_{\nu=0}^{j} \frac{\left.q^{(j-\nu}{ }^{2}\right)}{(q ; q)_{j-\nu}} \leq \frac{(-1 ; q)_{\infty}}{\left(\left|\eta / t_{1}\right| ; q\right)_{\infty}}
$$

holds for $\eta$ with $|\eta|<\left|t_{1}\right|$. In the last inequality we used Euler's identities (1.12) and (1.13). Hence for $|\eta|<\left|t_{1}\right|$, Tannery's theorem (the discrete version of the Lebesgue dominated convergence theorem) can be applied. Using formula (3.6) we get

$$
\begin{aligned}
\lim _{n \rightarrow \infty} \eta^{n} b_{n}(1 / \eta, \mathbf{t}) & =\sum_{j=0}^{\infty} \frac{q^{\left(\begin{array}{l}
j \\
2
\end{array}\right)\left(\eta / t_{1}\right)^{j}}}{(q ; q)_{j}} 3 \phi_{2}\left(\begin{array}{c}
q^{-j}, t_{1}, t_{2} \\
a t_{1} t_{2}, 0
\end{array} \mid q, q\right) \\
& =\sum_{k=0}^{\infty} \frac{\left(t_{1}, t_{2} ; q\right)_{k} q^{k}}{\left(a t_{1} t_{2}, q ; q\right)_{k}} \sum_{j=k}^{\infty} \frac{\left(q^{-j} ; q\right)_{k} q^{\left(\begin{array}{l}
j \\
2
\end{array}\right)\left(\eta / t_{1}\right)^{j}}}{(q ; q)_{j}} \\
& =\sum_{k=0}^{\infty} \frac{\left(t_{1}, t_{2} ; q\right)_{k}\left(-\eta / t_{1}\right)^{k}}{\left(a t_{1} t_{2}, q ; q\right)_{k}} \sum_{j=k}^{\infty} \frac{q^{\left(\begin{array}{c}
j-k \\
2
\end{array}\right)\left(\eta / t_{1}\right)^{j-k}}}{(q ; q)_{j-k}} \\
& =\left(-\eta / t_{1} ; q\right)_{\infty 2} \phi_{1}\left(\begin{array}{c}
t_{1}, t_{2} \\
a t_{1} t_{2}
\end{array} \mid q,-\eta / t_{1}\right),
\end{aligned}
$$

where we also used (1.13).

From (5.1), (5.5), and (5.4) for $z \notin \mathbf{R}$ we get

$$
\begin{aligned}
\int_{\mathbf{R}} \frac{d \varphi(u, \mathbf{t})}{z-u} & =\lim _{n \rightarrow \infty} \frac{z^{-n} q^{n-1} b_{n-1}(z / q, q \mathbf{t})}{z^{-n} b_{n}(z, \mathbf{t})} \\
& =\frac{{ }_{2} \phi_{1}\left(q t_{1}, q t_{2} ; a t_{1} t_{2} q^{2} ; q,-1 /\left(t_{1} z\right)\right)}{z_{2} \phi_{1}\left(t_{1}, t_{2} ; a t_{1} t_{2} ; q,-1 /\left(t_{1} z\right)\right)}, \quad|z|>1 /\left|t_{1}\right| .
\end{aligned}
$$


An analytic continuation of (5.6) can be found using the Heine transformation $[4,($ III.1)],

$$
{ }_{2} \phi_{1}(A, B ; C ; q, Z)=\frac{(B, A Z ; q)_{\infty}}{(C, Z ; q)_{\infty}} \phi_{1}(C / B, Z ; A Z ; q, B), \quad|Z|,|B|<1 .
$$

From (5.6) and (5.7) we obtain

$$
\int_{\mathbf{R}} \frac{d \varphi(u, \mathbf{t})}{z-u}=\frac{\left(1-a t_{1} t_{2}\right)\left(1-a t_{1} t_{2} q\right)}{\left(1-t_{2}\right)(z+1)} \frac{{ }_{2} \phi_{1}\left(a q t_{1},-1 /\left(t_{1} z\right) ;-q / z ; q, q t_{2}\right)}{{ }_{2} \phi_{1}\left(a t_{1},-1 /\left(t_{1} z\right) ;-1 / z ; q, t_{2}\right)} .
$$

The Heine transformation also provides an analytic continuation of formula (5.5).

Formulas (4.11)-(4.16), (4.22), and (5.5) imply

$$
\begin{aligned}
c_{0}(\lambda, \mathbf{t}) & =A((1-q) \lambda ; a, \mathbf{t})=((1-q) \lambda, a(1-q) \lambda ; q)_{\infty}^{-1} \\
& \times \sum_{j=0}^{\infty} \frac{q^{\left(\begin{array}{c}
j \\
2
\end{array}\right)(-a(1-q) \lambda)^{j}}}{(q ; q)_{j}}{ }_{3} \phi_{2}\left(q^{-j}, t_{1}, t_{2} ; a t_{1} t_{2}, 0 ; q, q\right) \\
& =\frac{1}{((1-q) \lambda ; q)_{\infty}} 2 \phi_{1}\left(\begin{array}{r}
t_{1}, t_{2} \\
a t_{1} t_{2}
\end{array} \mid q, a(1-q) \lambda\right) .
\end{aligned}
$$

From (5.6) and (5.9) we obtain

$$
\int_{\mathbf{R}} \frac{d \varphi(u, \mathbf{t})}{z-u}=\frac{c_{0}\left(-1 /\left(a(1-q) t_{1} z\right), q \mathbf{t}\right)}{z c_{0}\left(-1 /\left(a(1-q) t_{1} z\right), \mathbf{t}\right)} .
$$

The $c_{0}$-functions in (5.10) have no common zeros. This can be seen as follows: Assume that $c_{0}\left(\lambda_{0}, \mathbf{t}\right)=c_{0}\left(\lambda_{0}, q \mathbf{t}\right)=0$ for some $\lambda_{0}$. Formula (4.11) implies

$$
c_{n}(\lambda, q \mathbf{t})=\frac{\left(1-a t_{1} t_{2} q^{n}\right)\left(1-q^{n+1}\right) t_{1}}{\left(1-t_{1}\right)\left(1-a t_{1}\right)(1-q) \lambda} c_{n+1}(\lambda, \mathbf{t}) .
$$

Then our assumption implies $c_{1}\left(\lambda_{0}, \mathbf{t}\right)=0$ and from the three term recurrence equation (4.25) we get $c_{n}\left(\lambda_{0}, \mathbf{t}\right)=0$ for all $n \geq 0$. But then by Theorem 4.1,

$$
e_{q}\left(\lambda_{0}(1-q) x\right)=\left(\lambda_{0}(1-q) x ; q\right)_{\infty}^{-1}=\sum_{n=0}^{\infty} c_{n}\left(\lambda_{0}, \mathbf{t}\right) p_{n}(x, \mathbf{t}) \equiv 0,
$$

which is impossible.

The Perron-Stieltjes inversion formula is

$$
\begin{aligned}
F(z) & =\int_{\mathbf{R}} \frac{d \mu(t)}{z-t} \text { if and only if } \\
\mu(x)-\mu(y) & =\lim _{\epsilon \rightarrow 0^{+}} \int_{y}^{x} \frac{F(t-i \epsilon)-F(t+i \epsilon)}{2 \pi i} d t .
\end{aligned}
$$


This inversion formula shows that $\varphi$ is a purely discrete measure. It is clear that an isolated point mass $m$ of $\varphi$ located at $x=x_{0}$ contributes $m /\left(z-x_{0}\right)$ to the left-hand side of (5.8). Thus the isolated point masses of $\varphi$ coincide with the isolated poles of the right-hand side of (5.8) and the masses are the corresponding residues. Below we will show that $x=0$, which is the only essential singularity of the right-hand side of (5.8) does not support a discrete mass, so let us assume this for the time being. Formula (5.10), Theorem 4.2, and the above discussion describe the relationship between the support of the measure $\varphi(\cdot, \mathbf{t})$ and the spectrum of the operator $\mathcal{T}_{\mathbf{t}}$.

Theorem 5.1. The support of the measure of orthogonality $\varphi(\cdot, \mathbf{t})$ is the set of the elements of the spectrum of the operator $\mathcal{T}_{\mathbf{t}}$ multiplied by $-1 /(a(1-$ q) $\left.t_{1}\right)$.

It remains to show that $x=0$ is not a mass point for $\varphi$. From the theory of the moment problem [1], [14], it is known that when the measure $\varphi$ is unique then $x=x_{0}$ is a mass point for the measure if and only if $\sum_{n=0}^{\infty} p_{n}\left(x_{0}\right)^{2}$ converges, $\left\{p_{n}(x)\right\}$ being the orthonormal polynomials. From Theorem 3.1 we find

$b_{n}(0, \mathbf{t})=\frac{\left(q^{-n} / t_{1}, q^{-n} / t_{2} ; q\right)_{n}}{\left(q, q^{-2 n} /\left(a t_{1} t_{2}\right) ; q\right)_{n}}\left(-a t_{1}\right)^{-n} \phi_{3}\left(\begin{array}{c}q^{-n}, a t_{1} t_{2} q^{n+1}, t_{1}, t_{2} \\ t_{1} q, t_{2} q, a t_{1} t_{2}\end{array} \mid q, q\right)$.

Applying (1.15) to (5.12) we get

$$
b_{n}(0, \mathbf{t})=\frac{\left(q t_{1}, q t_{2} ; q\right)_{n}}{\left(q, a t_{1} t_{2} q^{n+1} ; q\right)_{n}} \frac{q^{\left(\begin{array}{c}
n \\
2
\end{array}\right)}}{t_{1}^{n}}{ }_{4} \phi_{3}\left(\begin{array}{c}
q^{-n}, a t_{1} t_{2} q^{n+1}, t_{1}, t_{2} \\
t_{1} q, t_{2} q, a t_{1} t_{2}
\end{array} \mid q, q\right) .
$$

Ismail and Wilson, [10], determined the asymptotic behavior of the AskeyWilson polynomials. They proved that

$$
\begin{aligned}
& \lim _{n \rightarrow \infty}(z / A)^{n}{ }_{4} \phi_{3}\left(\begin{array}{c}
q^{-n}, A B C D q^{n-1}, A z, A / z \\
A B, A C, A D
\end{array} \mid q, q\right) \\
& =\frac{(A z, B z, C z, D z ; q)_{\infty}}{\left(z^{2}, A B, A C, A D ; q\right)_{\infty}},
\end{aligned}
$$

for $|z|<1$, and that the left-hand side of (5.14) is bounded if $|z|=1$ but $z \neq \pm 1$. If $z= \pm 1$ then the left-hand side of (5.14) is $O(n)$. The ${ }_{4} \phi_{3}$ quantity in (5.13) corresponds to the ${ }_{4} \phi_{3}$ function in (5.14) with $A=\sqrt{t_{1} t_{2}}$, $B=q \sqrt{t_{1} / t_{2}}, C=q \sqrt{t_{2} / t_{1}}, D=a \sqrt{t_{1} t_{2}}$, and $z=\sqrt{t_{1} / t_{2}}$ if $\left|t_{1}\right| \leq\left|t_{2}\right|$ and $z=\sqrt{t_{2} / t_{1}}$ otherwise. The orthonormal polynomials associated with the $b_{n}$ 's are

$$
p_{m}(\eta, \mathbf{t})=b_{m}(\eta, \mathbf{t}) \xi_{0}^{-1 / 2}(\mathbf{t}) \prod_{n=1}^{m} u_{n}^{-1 / 2}
$$


where $-u_{n}$ denotes the coefficient of $b_{n-1}(\eta, \mathbf{t})$ in (2.18). From (2.18) we get

$$
\prod_{k=1}^{n} u_{k}=\frac{\left(q t_{1}, a q t_{1}, q t_{2}, a q t_{2} ; q\right)_{n}}{\left(a t_{1} t_{2} q, a t_{1} t_{2} q^{2} ; q\right)_{2 n}}\left(t_{2} / t_{1}\right)^{n} q^{2\left(\begin{array}{c}
n+1 \\
2
\end{array}\right)-n} .
$$

Combining the above formulas we obtain

$$
\begin{aligned}
q^{n} \xi_{0}(\mathbf{t}) z^{2 n} p_{n}(0, \mathbf{t})^{2} & =\frac{\left(q t_{1}, q t_{2} ; q\right)_{n}}{\left(a q t_{1}, a q t_{2} ; q\right)_{n}} \frac{\left(a t_{1} t_{2} q ; q\right)_{n}^{2}\left(1-a t_{1} t_{2} q^{2 n+1}\right)}{(q ; q)_{n}^{2}\left(1-a t_{1} t_{2} q\right)} \frac{z^{2 n}}{\left(t_{1} t_{2}\right)^{n}} \\
& \times{ }_{4} \phi_{3}\left(\begin{array}{c}
q^{-n}, a t_{1} t_{2} q^{n+1}, t_{1}, t_{2} \\
q t_{1}, q t_{2}, a t_{1} t_{2}
\end{array} \mid q, q\right)^{2} \\
& \rightarrow \frac{\left(q t_{1}, q t_{2} ; q\right)_{\infty}\left(a t_{1} t_{2} q ; q\right)_{\infty}^{2}}{\left(a q t_{1}, a q t_{2} ; q\right)_{\infty}\left(1-a t_{1} t_{2} q\right)} \\
& \times \frac{\left(z \sqrt{t_{1} t_{2}}, q z \sqrt{t_{1} / t_{2}}, q z \sqrt{t_{2} / t_{1}}, a z \sqrt{t_{1} t_{2}} ; q\right)_{\infty}^{2}}{\left(z^{2}, q t_{1}, q t_{2}, a t_{1} t_{2}, q ; q\right)_{\infty}^{2}}, \quad n \rightarrow \infty,
\end{aligned}
$$

if $|z| \leq 1$ and $z \neq \pm 1$. If $z=1$ the right-hand side of (5.15) becomes unbounded. Since $|z| \leq 1$ and $|q|<1,(5.15)$ clearly implies that $\sum_{n=0}^{\infty} p_{n}(0, \mathbf{t})^{2}$ diverges.

Equations (3.1)-(3.3) show that the polynomials $\left\{b_{n}(\eta, \mathbf{t})\right\}$ are constant multiples of birth and death process polynomials associated with a process with birth and death rates

$$
\frac{\left(1-t_{1} q^{n+1}\right)\left(1-a t_{1} q^{n+1}\right) t_{2} q^{n}}{\left(1-a t_{1} t_{2} q^{2 n+1}\right)\left(1-a t_{1} t_{2} q^{2 n+2}\right)} \quad \text { and } \quad \frac{\left(1-t_{2} q^{n}\right)\left(1-a t_{2} q^{n}\right) t_{1} q^{n}}{\left(1-a t_{1} t_{2} q^{2 n}\right)\left(1-a t_{1} t_{2} q^{2 n+1}\right)}
$$

respectively. An exposition of the theory of birth and death processes and orthogonal polynomials can be found in [7].

\section{Connection Coefficients for the Big $q$-Jacobi Polynomials.}

In this section we will compute the connection coefficients in the formula

$$
p_{n}(x, \mathbf{t})=\sum_{l=0}^{n} a_{n, l}(\mathbf{t}, \mathbf{s}) p_{l}(x, \mathbf{s})
$$


where $\mathbf{t}=\left(t_{1}, t_{2}\right)$ and $\mathbf{s}=\left(s_{1}, s_{2}\right)$. From (6.1), (1.6), and (1.10) we get

$$
\begin{aligned}
& a_{n, l}(\mathbf{t}, \mathbf{s}) \xi_{l}(\mathbf{s}) \\
& =\int_{\mathbf{R}} p_{n}(x, \mathbf{t}) p_{l}(x, \mathbf{s}) d \mu(x, \mathbf{s}) \\
& =\int_{\mathbf{R}} \sum_{k=0}^{n} \frac{\left(q^{-n}, a t_{1} t_{2} q^{n-1} ; q\right)_{k} q^{k}}{\left(t_{1}, a t_{1}, q ; q\right)_{k}}\left(\left(t_{1} / s_{2} ; q\right)_{k} \sum_{\nu=0}^{k} \frac{\left(q^{-k}, s_{2} x ; q\right)_{\nu} q^{\nu}}{\left(s_{2} q^{1-k} / t_{1}, q ; q\right)_{\nu}}\right) \\
& \quad \times \sum_{j=0}^{l} \frac{\left(q^{-l}, a s_{1} s_{2} q^{l-1} ; q\right)_{j} q^{j}}{\left(s_{1}, a s_{1}, q ; q\right)_{j}}\left(s_{1} x ; q\right)_{j} d \mu(x, \mathbf{s}) .
\end{aligned}
$$

Changing the order of summation in (6.2) we obtain

$$
\begin{aligned}
a_{n, l}(\mathbf{t}, \mathbf{s}) \xi_{l}(\mathbf{s})= & \sum_{\nu=0}^{n}\left(\sum_{k=\nu}^{n} \frac{\left(q^{-n}, a t_{1} t_{2} q^{n-1} ; q\right)_{k} q^{k}}{\left(t_{1}, a t_{1}, q ; q\right)_{k}} \frac{\left(t_{1} / s_{2} ; q\right)_{k}\left(q^{-k} ; q\right)_{\nu}}{\left(s_{2} q^{1-k} / t_{1} ; q\right)_{\nu}}\right) \frac{q^{\nu}}{(q ; q)_{\nu}} \\
& \times \sum_{j=0}^{l} \frac{\left(q^{-l}, a s_{1} s_{2} q^{l-1} ; q\right)_{j} q^{j}}{\left(s_{1}, a s_{1}, q ; q\right)_{j}} \int_{\mathbf{R}} \frac{d \mu^{(a)}(x)}{\left(x s_{1} q^{j}, x s_{2} q^{\nu} ; q\right)_{\infty}} .
\end{aligned}
$$

The last integral is evaluated using the $q$-beta integral evaluation from [6]

$$
\int_{\mathbf{R}} \frac{d \mu^{(a)}(x)}{\left(x t_{1}, x t_{2} ; q\right)_{\infty}}=\frac{\left(a t_{1} t_{2} ; q\right)_{\infty}}{\left(t_{1}, a t_{1}, t_{2}, a t_{2} ; q\right)_{\infty}} .
$$

To evaluate the last sum in (6.3) we use (1.9). We get

$$
\begin{aligned}
& \frac{\left(a s_{1} s_{2} q^{\nu} ; q\right)_{\infty}}{\left(s_{1}, a s_{1}, s_{2} q^{\nu}, a s_{2} q^{\nu} ; q\right)_{\infty}} \sum_{j=0}^{l} \frac{\left(q^{-l}, a s_{1} s_{2} q^{l-1} ; q\right)_{j}}{\left(a s_{1} s_{2} q^{\nu}, q ; q\right)_{j}} q^{j} \\
& =\frac{\left(a s_{1} s_{2} q^{\nu} ; q\right)_{\infty}}{\left(s_{1}, a s_{1}, s_{2} q^{\nu}, a s_{2} q^{\nu} ; q\right)_{\infty}} \frac{\left(q^{\nu+1-l} ; q\right)_{l}}{\left(a s_{1} s_{2} q^{\nu} ; q\right)_{l}}\left(a s_{1} s_{2} q^{l-1}\right)^{l}
\end{aligned}
$$

Since $\left(q^{\nu+1-l} ; q\right)_{l}$ vanishes for $\nu<l$, the first sum in (6.3) is over $\nu \in$ $\{l, \ldots, n\}$. 
Let $S_{n, \nu}(\mathbf{t}, \mathbf{s})$ denote the sum over $k$ in equation (6.3). Applying (1.15) to $\left(q^{-k} ; q\right)_{\nu}$ and $\left(s_{2} q^{1-k} / t_{1} ; q\right)_{\nu}$ we obtain

$$
\begin{gathered}
S_{n, \nu}(\mathbf{t}, \mathbf{s})=\frac{\left(q^{-n}, a t_{1} t_{2} q^{n-1} ; q\right)_{\nu} q^{\nu}}{\left(t_{1}, a t_{1} ; q\right)_{\nu}} \\
\times \sum_{k=\nu}^{n} \frac{\left(q^{-(n-\nu)}, a t_{1} t_{2} q^{n+\nu-1} ; q\right)_{k-\nu} q^{k-\nu}}{\left(t_{1} q^{\nu}, a t_{1} q^{\nu} ; q\right)_{k-\nu}} \frac{q^{-\nu}\left(t_{1} / s_{2} ; q\right)_{k-\nu}}{\left(s_{2} / t_{1}\right)^{\nu}(q ; q)_{k-\nu}} \\
=\frac{\left(q^{-n}, a t_{1} t_{2} q^{n-1} ; q\right)_{\nu}\left(t_{1} / s_{2}\right)^{\nu}}{\left(t_{1}, a t_{1} ; q\right)_{\nu}}{ }_{3} \phi_{2}\left(\begin{array}{c}
q^{-(n-\nu)}, a t_{1} t_{2} q^{n+\nu-1}, t_{1} / s_{2} \\
t_{1} q^{\nu}, a t_{1} q^{\nu}
\end{array} \mid q, q\right) .
\end{gathered}
$$

From (6.3), (6.5), and (6.6) using the identities $\left(q^{\nu+1-l} ; q\right)_{l} /(q ; q)_{\nu}=$ $1 /(q ; q)_{\nu-l}$ and

$$
\frac{\left(a s_{1} s_{2} q^{\nu} ; q\right)_{\infty}}{\left(a s_{1} s_{2} q^{\nu} ; q\right)_{l}}=\left(a s_{1} s_{2} q^{\nu+l} ; q\right)_{\infty}=\frac{\left(a s_{1} s_{2} q^{2 l} ; q\right)_{\infty}}{\left(a s_{1} s_{2} q^{2 l} ; q\right)_{\nu-l}}
$$

we obtain the following result.

Theorem 6.1. The connection coefficients in the expansion of the polynomial $p_{n}(x, \mathbf{t})$ in terms of the polynomials $\left\{p_{l}(x, \mathbf{s})\right\}$ are given by the formula

$$
\begin{aligned}
a_{n, l}(\mathbf{t}, \mathbf{s})= & \frac{1}{\xi_{l}(\mathbf{s})} \frac{\left(a s_{1} s_{2} q^{2 l} ; q\right)_{\infty}\left(a t_{1} s_{1} q^{l}\right)^{l}}{\left(s_{1}, a s_{1}, s_{2} q^{l}, a s_{2} q^{l} ; q\right)_{\infty}} \frac{\left(q^{-n}, a t_{1} t_{2} q^{n-1} ; q\right)_{l}}{\left(t_{1}, a t_{1} ; q\right)_{l}} \\
& \times \sum_{\nu=l}^{n} \frac{\left(q^{-(n-l)}, a t_{1} t_{2} q^{n+l-1}, s_{2} q^{l}, a s_{2} q^{l} ; q\right)_{\nu-l}\left(t_{1} q / s_{2}\right)^{\nu-l}}{\left(t_{1} q^{l}, a t_{1} q^{l}, a s_{1} s_{2} q^{2 l}, q ; q\right)_{\nu-l}} \\
& \times{ }_{3} \phi_{2}\left(\begin{array}{c}
q^{-(n-\nu)}, a t_{1} t_{2} q^{n+\nu-1}, t_{1} / s_{2} \\
t_{1} q^{\nu}, a t_{1} q^{\nu}
\end{array} \mid q, q\right), \quad l=0, \ldots, n .
\end{aligned}
$$

The connection coefficient formula (6.7) can be used to find the connection coefficients in certain special cases. In Section 2, we computed these coefficients for the case $\mathbf{s}=q \mathbf{t}$.

We will now use (6.7) to give another proof of the fact that $a_{n, l}(\mathbf{t}, q \mathbf{t})=$ 0 for $l<n-2$. In view of (1.8) it is enough to consider the case $\mathbf{s}=$ $\left(q t_{2}, q t_{1}\right)$ since for every $l \in \mathbf{N}, p_{l}\left(x ; t_{1}, t_{2}\right)$ and $p_{l}\left(x ; t_{2}, t_{1}\right)$, and therefore the coefficients $a_{n, l}(\mathbf{t}, q \mathbf{t})$ and $a_{n, l}\left(\mathbf{t}, q \mathbf{t}^{*}\right)$ are linearly dependent, where $\mathbf{t}^{*}=$ $\left(t_{2}, t_{1}\right)$. So let $\mathbf{s}=q \mathbf{t}^{*}$. In view of (6.7) it is enough to show that $\tilde{S}_{n, l}(\mathbf{t})=0$ for $l<n-2$, where $\tilde{S}_{n, l}(\mathbf{t})$ denotes the sum in (6.7) with $\mathbf{s}=q \mathbf{t}^{*}$. For this 
sum by (6.7) we have

$$
\begin{gathered}
\left(1-t_{1} q^{l}\right)\left(1-a t_{1} q^{l}\right) \tilde{S}_{n, l}(\mathbf{t})=\sum_{\nu=l}^{n} \frac{\left(q^{-(n-l)}, a t_{1} t_{2} q^{n+l-1} ; q\right)_{\nu-l}}{\left(a t_{1} t_{2} q^{2 l+2}, q ; q\right)_{\nu-l}} \\
\times\left(1-t_{1} q^{\nu}\right)\left(1-a t_{1} q^{\nu}\right)\left(1-\frac{\left(1-q^{-(n-\nu)}\right)\left(1-a t_{1} t_{2} q^{n+\nu-1}\right)}{\left(1-t_{1} q^{\nu}\right)\left(1-a t_{1} q^{\nu}\right)}\right) \\
=\sum_{i=0}^{n-l} \frac{\left(q^{-(n-l)}, a t_{1} t_{2} q^{n+l-1} ; q\right)_{i}}{\left(a t_{1} t_{2} q^{2 l+2}, q ; q\right)_{i}} \\
\times\left[\left(1-t_{1} q^{l+i}\right)\left(1-a t_{1} q^{l+i}\right)-\left(1-q^{-(n-l)+i}\right)\left(1-a t_{1} t_{2} q^{n+l+i-1}\right)\right] \\
=q^{l}\left(-t_{1}-a t_{1}+q^{-n}+a t_{1} t_{2} q^{n-1}\right)_{2} \phi_{1}\left(\begin{array}{c}
q^{-d}, a t_{1} t_{2} q^{d+2 l-1} \\
a t_{1} t_{2} q^{2 l+2}
\end{array} \mid q, q\right) \\
+\left(a t_{1}^{2} q^{2 l}-a t_{1} t_{2} q^{2 l-1}\right)_{2} \phi_{1}\left(\begin{array}{c}
q^{-d}, a t_{1} t_{2} q^{d+2 l-1} \\
a t_{1} t_{2} q^{2 l+2}
\end{array} \mid q, q^{2}\right),
\end{gathered}
$$

with $d=n-l$. We now show that the ${ }_{2} \phi_{1}$ expressions in (6.8) vanish.

For integer numbers $d>0, d_{1} \geq 0$, and $d_{2}>0$ we consider

$$
A_{d, d_{1}, d_{2}}(\alpha, q):={ }_{2} \phi_{1}\left(\begin{array}{c|c}
q^{-d}, \alpha q^{d_{1}} & q, q^{d_{2}} \\
\alpha & .
\end{array}\right.
$$

We claim that $A_{d, d_{1}, d_{2}}(\alpha, q) \equiv 0$ if $d_{1}+d_{2}<d$. Indeed we have

$$
\begin{aligned}
A_{d, d_{1}, d_{2}}(\alpha, q) & =\sum_{j=0}^{d} \frac{\left(q^{-d}, \alpha q^{d_{1}} ; q\right)_{j}}{(\alpha, q ; q)_{j}} q^{d_{2} j} \\
& =\sum_{j=0}^{d}\left[\begin{array}{l}
d \\
j
\end{array}\right]_{q} \frac{\left(\alpha q^{d_{1}} ; q\right)_{j}}{(\alpha ; q)_{j}}\left(-q^{-d+d_{2}}\right)^{j} q^{\left(\begin{array}{c}
j \\
2
\end{array}\right)} \\
& =\sum_{j=0}^{d}\left[\begin{array}{l}
d \\
j
\end{array}\right]_{q} \frac{\left(\alpha q^{j} ; q\right)_{d_{1}}}{(\alpha ; q)_{d_{1}}}\left(-q^{-\left(d-d_{2}\right)}\right)^{j} q^{\left(\begin{array}{c}
j \\
2
\end{array}\right)}
\end{aligned}
$$

where we used the identity

$$
\left(\alpha q^{j} ; q\right)_{d_{1}}(\alpha ; q)_{j}=(\alpha ; q)_{d_{1}+j}=\left(\alpha q^{d_{1}} ; q\right)_{j}(\alpha ; q)_{d_{1}}
$$


By the $q$-binomial theorem $(1.14)$ we have $(z ; q)_{l}=\sum_{s=0}^{l} a_{l, s}(q) z^{s}$ with coefficients $a_{l, s}(q)=(-1)^{s} q^{\left(\begin{array}{c}s \\ 2\end{array}\right)}\left[\begin{array}{l}l \\ s\end{array}\right]_{q}$. Then $(6.10)$ can be continued as follows

$$
\begin{aligned}
A_{d, d_{1}, d_{2}}(\alpha, q) & =\frac{1}{(\alpha ; q)_{d_{1}}} \sum_{s=0}^{d_{1}} a_{d_{1}, s}(q) \alpha^{s}\left(\sum_{j=0}^{d}\left[\begin{array}{l}
d \\
j
\end{array}\right]_{q}\left(-q^{s-\left(d-d_{2}\right)}\right)^{j} q^{\left(\begin{array}{c}
j \\
2
\end{array}\right)}\right) \\
& =\left(1 /(\alpha ; q)_{d_{1}}\right) \sum_{s=0}^{d_{1}} a_{d_{1}, s}(q) \alpha^{s}\left(q^{s-\left(d-d_{2}\right)} ; q\right)_{d}=0, \quad d-d_{2}>d_{1},
\end{aligned}
$$

where at the end we used (1.14). From (6.8) and (6.11) we get $\tilde{S}_{n, l}(\mathbf{t})=0$ for $d=n-l>2$, since in this case it is a linear combination of $A_{d, d-3,1}(\alpha, q)$ and $A_{d, d-3,2}(\alpha, q)$ with $\alpha=a t_{1} t_{2} q^{2 l+2}$. Then $a_{n, l}(\mathbf{t}, q \mathbf{t})=0$ for $l<n-2$.

\section{References}

[1] N.I. Akhiezer, The Classical Moment Problem and Some Related Questions in Analysis, Oliver and Boyd, Edinburgh, 1965 (English translation).

[2] G.E. Andrews and R.A. Askey, Classical Orthogonal Polynomials, Polynomes Orthogonaux et Applications (C. Breziniski et al., eds.), Lecture Notes in Mathematics, 1171, Springer-Verlag, Berlin, (1984), 36-63.

[3] G.E. Andrews, R.A. Askey and R. Roy, Special Functions, Cambridge University Press, Cambridge, 1999.

[4] G. Gasper and M. Rahman, Basic Hypergeometric Series, Cambridge University Press, Cambridge, 1989.

[5] D.P. Gupta, M.E.H. Ismail and D.R. Masson, Contiguous relations, basic hypergeometric functions, and orthogonal polynomials, II: Associated big q-Jacobi polynomials, J. Math. Anal. Appl., 171 (1992), 477-497.

[6] M.E.H. Ismail, Continuous and Discrete q-Orthogonal Polynomials, Monographmanuscript, 1998.

[7] M.E.H. Ismail, J. Letessier, D.R. Masson and G. Valent, Birth and Death Processes and Orthogonal Polynomials, Orthogonal Polynomials: Theory and Practice (P. Nevai, ed.), NATO, ASI on Orthogonal Polynomials and Their Applications, Kluwer Academic Publishers, (1990), 225-229.

[8] M.E.H. Ismail and M. Rahman, The associated Askey-Wilson polynomials, Trans. Amer. Math. Soc., 328 (1991), 201-237.

[9] M.E.H. Ismail, M. Rahman and R. Zhang, Diagonalization of certain integral operators II, J. Comp. Applied Math., 68 (1996), 163-196.

[10] M.E.H. Ismail and J.A. Wilson, Asymptotic and generating relations for the $q$-Jacobi and ${ }_{4} \phi_{3}$ polynomials, J. Approx. Theory, 36 (1982), 43-54.

[11] M.E.H. Ismail and R. Zhang, Diagonalization of certain integral operators, Adv. Math., 109 (1994), 1-33. 
[12] R. Koekoek and R. Swarttouw, The Askey-scheme of Hypergeometric Orthogonal Polynomials and its q-analogue, Technical Report, 94-05, Delft University of Technology, Delft, The Netherlands, 1994.

[13] H.M. Schwartz, A class of continued fractions, Duke Math. J., 6 (1940), 48-65.

[14] J. Shohat and J.D. Tamarkin, The Problem of Moments (Revised edition), Amer. Math. Soc., Providence, RI, 1950.

[15] G. Szegö, Orthogonal Polynomials, Amer. Math. Soc., Providence, RI, 4th edition, 1975 .

[16] J. Wimp, Some explicit Padé approximants for the function $\phi^{\prime} / \phi$ and a related quadrature formula involving Bessel functions, SIAM J. Math. Anal., 16 (1985), 887-895.

Received May 14, 1999. The first author's research partially was supported by NSF grant DMS 99-70865.

Department of Mathematics

UNIVERSITY OF SOUTH FLORIDA

TAMPA, FL 33620-5700

E-mail address: ismail@math.usf.edu

Department of Mathematics \& Computer Science

UNIVERSITY OF HOUSTON-DOWNTOWN

Houston, TX 77002-1094

E-mail address: simeonovp@zeus.dt.uh.edu 